\title{
A conservative strategy to couple 1D and 2D models for shallow water flow simulation
}

\author{
M. Morales-Hernández*, P. García-Navarro*, J. Burguete**, P. Brufau*
}

\begin{abstract}
A 1D-2D coupled numerical model is presented in this work. $1 \mathrm{D}$ and 2D models are formulated using a conservative upwind cell-centered finite volume scheme. The discretization is based on cross sections for the 1D model and with triangular unstructured grid for the 2D model. The resulting element of discretization for the coupled model is analysed and two different coupling techniques based on mass conservation and mass and momentum conservation respectively are explored, considering both frontal and lateral configurations. The interaction with the boundaries in each model is highlighted and the necessity of using the appropriate strategy according to the flow regime is also justified. The coupled model is tested through academic test cases where the numerical results are compared with a fully $2 \mathrm{D}$ model as well as with experimental measurements in steady and unsteady scena-
\end{abstract}

\footnotetext{
${ }^{*}$ Fluid Mechanics, LIFTEC, EINA, Universidad Zaragoza C/Maria de Luna,3 50018 Zaragoza, Spain

**Dept. Soil and Water, Aula Dei Experimental Stn., CSIC, P.O. Box 202, 50080 Zaragoza, Spain. BIFI, Universidad Zaragoza

Email addresses: mmorales@unizar.es (M. Morales-Hernández), pigar@unizar.es (P. García-Navarro), jburguete@eead.csic.es (J. Burguete), brufau@unizar .es (P. Brufau)
} 
rios. It is also applied to a real world configuration, where the flood wave propagation in the river bed is simulated by means of a $1 \mathrm{D}$ model and the inundation of the riverside is dealt with a $2 \mathrm{D}$ model. The computational gain is also analysed.

Keywords: 1D-2D coupled model, shallow water flows, numerical modelling, conservation, boundary conditions

\section{Introduction}

2 Growing population and economic activities near rivers have caused an 3 increased flood risk to many urban regions. Computers and modelization 4 help assess and manage flood risk. One dimensional (1D) hydrodynamic 5 models have been widely used in modelling flood flows $[1,2,3]$. This type 6 of models are computationally efficient for dealing with large river/channel 7 systems and several other hydraulic structures. However, when modelling 8 floodplain flows, their accuracy and appropriateness decreases. Quasi 2D 9 models have been developed for that situation, in which the floodplain is discretized into a network of virtual river branches and spills linked with 11 main river channels $[4,5,6]$. Although this approach has been successfully used for many flood studies, it is generally time-consuming in setting up the initial model and the accuracy of predictions varies with the way in which 14 the floodplain is discretized. Depth-integrated two dimensional (2D) hydro15 dynamic models have been used for many years for predicting free surface 
with channel networks and hydraulic structures. The increasing availability of digital topographic data in recent years provides this type of models with a wider scope of application. 1D approximations require less information and are computationally time saving while 2D models when the real flow pattern does not correspond with a $1 \mathrm{D}$ domain, give more precise results but are time consuming and more topographical demanding. Therefore, with the need to improve modelling accuracy and to gain computational time, coupled modelling approaches of $1 \mathrm{D}$ and $2 \mathrm{D}$ shallow water models are increasingly used.

Coupled 1D-2D models have been developed in recent years and successfully applied to large and complex river systems $[7,8,9,10]$. Some authors $[11,12]$ propose using only the $1 \mathrm{D}$ model to predict flow velocity and water level within the main river network. If large areas are inundated owing to a breach of a section of river embankment, it is likely that the flows would no longer be 1D. In such case the 2D model is used to predict the flow velocity and inundation levels in the flooded area. The models are linked by a weir equation, in which the volume of flow from the $1 \mathrm{D}$ domain to the $2 \mathrm{D}$ domain is determined by the water level difference. Another form to couple 1D-2D hydrodynamic models consists of a transformation of $2 \mathrm{D}$ quantities to $1 \mathrm{D}$ quantities just averaging the $2 \mathrm{D}$ terms along the cross sections and imposing continuity at the interfaces. After that, a subdomain iterative procedure is carried out to solve the coupled 1D-2D problem [13]. This technique turns out to be a reliable strategy provided that a proper choice of the subdomain 
40

is performed, only for simple configurations (e.g. a straight channel or a river bifurcation). Some recent works propose more sophisticated ways for 'stitching' both models. For example, they can be connected by internally coupling the 1D node with the center of the 2D grid cell [14], by considering the numerical fluxes of each model [15] and also by introducing several corrections in the momentum quantity transfer due to the occurrence of swirls [16]. The concern of source terms and the possibility of linking both models in discontinuous topography is explored in [17].

Most of these coupled model approaches, developed from previous existing $1 \mathrm{D}$ and 2D models, require a deeply overview concerning how each model is perceiving the coupling by itself. The boundary conditions in each model play an important role within the modelization due to the fact that the end of the $2 \mathrm{D}$ domain is always interacting with the $1 \mathrm{D}$ model hence the boundary treatment should be continuously considered.

Bearing this in mind, two coupling strategies based on a mass conservation and a complete mass and momentum conservation will be proposed in order to cover all possible flow situations and to approximate faithfully the results given by a fully $2 \mathrm{D}$ model. The formulation is presented in a general expression, covering both frontal and lateral coupling configurations with respect to the $1 \mathrm{D}$ model. The bed slope and friction source terms relating to the $1 \mathrm{D}$ and the $2 \mathrm{D}$ models are included in the formulation of the coupled scheme. Emphasizing the idea of a correct conservation philosophy and taking into account the information which leaves out the $1 \mathrm{D}$ or $2 \mathrm{D}$ 
domains, the adequate use of each strategy according to the flow conditions will be inherently justified and subsequently corroborated. Both models built using a conservative upwind cell-centered finite volume scheme based on Roe Riemann solver across the edges [18]. The topography is represented with cross sections for the 1D model and with DTM (Digital Terrain Model) in a triangular unstructured grid for the 2D model.

The main objective of this manuscript is to enhance the correct formulation of coupled models based on existing 1D and 2D models. One test has been chosen for calibration corresponding to a extreme dam break in a channel propagating into a flood plain [19]. Being a test case without almost influence of source terms, the hydrodynamic of the system can be deeply analysed when coupling both models. Then, a trapezoidal channel connected laterally with a floodplain area is used as validation test case including steady and unsteady flow scenarios and comparing the numerical results with a fully $2 \mathrm{D}$ model in terms of time evolution of several probes located at the domain. The behaviour of this coupled model is also performed in a Y-shape junction problem, with two geometry configurations that have an impact on the flow regime. Finally, it is applied to the Ebro river, a real meandering river with complex topography where the numerical results of the coupled model in terms of flooding extension and longitudinal profiles are compared with those obtained with a fully 2D modelization. The computational gain achieved by the proposed 1D-2D coupled model is also estimated in all the test cases presented, analysing the results in terms of 
speed-up in comparison with a complete 2D model.

\section{Governing equations}

88

89 90

$$
\begin{gathered}
\frac{\partial \mathbf{U}(x, t)}{\partial t}+\frac{d \mathbf{F}(x, \mathbf{U})}{d x}=\mathbf{H}(x, \mathbf{U}) \\
\mathbf{U}=\left(\begin{array}{c}
A \\
Q
\end{array}\right), \quad \mathbf{F}=\left(\begin{array}{c}
Q \\
\frac{Q^{2}}{A}+g I_{1}
\end{array}\right), \quad \mathbf{H}=\left(\begin{array}{c}
0 \\
g\left[I_{2}+A\left(S_{0}-S_{f}\right)\right]
\end{array}\right)
\end{gathered}
$$

91 where $Q$ is the discharge, $A$ is the wetted cross section area, $g$ is the acceler92 ation due to the gravity, $S_{0}$ is the bed slope

$$
S_{0}=-\frac{\partial z_{b}}{\partial x}
$$

${ }_{93}$ where $z_{b}$ is the bed level. $S_{f}$ is the friction slope here represented by the 94 empirical Manning law

$$
S_{f}=\frac{Q^{2} n^{2}}{A^{2} R^{4 / 3}}
$$

${ }_{95}$ being $R$ the hydraulic radius and $n$ the Manning's roughness coefficient. $I_{1}$ 96 represents a hydrostatic pressure force term 


$$
I_{1}(x)=\int_{0}^{h}(h-\eta) \sigma(x, \eta) d \eta
$$

97

99 sectional wet area can be expressed as follows:

$$
A(x)=\int_{0}^{h} \sigma(x, \eta) d \eta
$$

100

On the other hand, $I_{2}$ accounts for the pressure force due to the longitudinal width variations:

$$
I_{2}(x)=\int_{0}^{h}(h-\eta) \frac{\partial \sigma(x, \eta)}{\partial x} d \eta
$$

$$
\mathbf{U}=\left(h, q_{x}, q_{y}\right)^{T}
$$

where the conserved variables:

$q_{x}=u h$ and $q_{y}=v h$, and the fluxes of these variables:

$$
\frac{\partial \mathbf{U}}{\partial t}+\frac{\partial \mathbf{F}_{x}(\mathbf{U})}{\partial x}+\frac{\partial \mathbf{F}_{y}(\mathbf{U})}{\partial y}=\mathbf{H}(\mathbf{U})
$$




$$
\mathbf{F}_{x}=\left(q_{x}, \frac{q_{x}^{2}}{h}+\frac{1}{2} g h^{2}, \frac{q_{x} q_{y}}{h}\right)^{T}, \quad \mathbf{F}_{y}=\left(q_{y}, \frac{q_{x} q_{y}}{h}, \frac{q_{y}^{2}}{h}+\frac{1}{2} g h^{2}\right)^{T}
$$

$$
\mathbf{H}=\left(0, g h\left(S_{0 x}-S_{f x}\right), g h\left(S_{0 y}-S_{f y}\right)\right)^{T}
$$

where the bed slopes of the bottom level $z_{b}$ are

$$
S_{0 x}=-\frac{\partial z_{b}}{\partial x}, \quad S_{0 y}=-\frac{\partial z_{b}}{\partial y}
$$

and the friction losses are written in terms of the Manning's roughness coefficient $n$ :

$$
S_{f x}=\frac{n^{2} u \sqrt{u^{2}+v^{2}}}{h^{4 / 3}}, \quad S_{f y}=\frac{n^{2} v \sqrt{u^{2}+v^{2}}}{h^{4 / 3}}
$$

\section{Numerical scheme}

The numerical scheme applied in this work is the first order upwind finite volume model. In both $1 \mathrm{D}$ and $2 \mathrm{D}$ cases, the system can be written:

$$
\frac{\partial \mathbf{U}}{\partial t}+\vec{\nabla} \mathbf{E}=\mathbf{H}
$$

being $\mathbf{E}=\mathbf{F}$ in the $1 \mathrm{D}$ model and $\mathbf{E}=\left(\mathbf{F}_{x}, \mathbf{F}_{y}\right)$ in the $2 \mathrm{D}$ case. Integrated in a volume or grid cell $\Omega$ : 
$\frac{\partial}{\partial t} \int_{\Omega} \mathbf{U} d \Omega+\int_{\Omega} \vec{\nabla} \mathbf{E} d \Omega=\int_{\Omega} \mathbf{H} d \Omega \Rightarrow \frac{\partial}{\partial t} \int_{\Omega} \mathbf{U} d \Omega+\oint_{S} \mathbf{E} \cdot \mathbf{n} d S=\int_{\Omega} \mathbf{H} d \Omega$

115 where $\mathbf{n}$ is the outward normal direction, $\mathbf{E} \cdot \mathbf{n}$ is the normal flux and $S$ 116 denotes the surface surrounding the volume $\Omega$.

\subsection{D numerical scheme}

${ }_{118}$ It is possible to express the equations (1), (2) in a non-conservative form 119 as in [20]:

$$
\frac{d \mathbf{F}(x, \mathbf{U})}{d x}=\left.\frac{\partial \mathbf{F}(x, \mathbf{U})}{\partial x}\right|_{\mathbf{U}=\text { const }}+\left.\frac{\partial \mathbf{F}(x, \mathbf{U})}{\partial \mathbf{U}}\right|_{x=\text { const }} \frac{\partial \mathbf{U}(x, t)}{\partial x}
$$

Using (16), the 1D shallow water equations can be formulated as follows :

$$
\frac{\partial \mathbf{U}(x, t)}{\partial t}+\mathbf{J}(x, \mathbf{U}) \frac{\partial \mathbf{U}(x, t)}{\partial x}=\mathbf{H}^{\prime}(x, \mathbf{U})
$$

121

being $\mathbf{H}^{\prime}(x, \mathbf{U})$ the vector related with the sources expressed in the nonconservative form:

$$
\mathbf{H}^{\prime}(x, \mathbf{U})=\mathbf{H}(x, \mathbf{U})-\frac{\partial \mathbf{F}(x, \mathbf{U})}{\partial x}
$$

and $\mathbf{J}$ the Jacobian matrix of the original system 


$$
\mathbf{J}=\frac{\partial \mathbf{F}}{\partial \mathbf{U}}=\left(\begin{array}{cc}
0 & 1 \\
c^{2}-u^{2} & 2 u
\end{array}\right)
$$

124 with $u=Q / A$ and $c=\sqrt{g A / B}$ ( $B$ is the top width at the free surface).

Following the Leibnitz rule, it is possible to express the link between $I_{1}$ and $I_{2}$ in this manner [21]:

$$
\frac{\partial I_{1}}{\partial x}=I_{2}+A \frac{\partial h}{\partial x}
$$

$$
\mathbf{H}^{\prime}(x, \mathbf{U})=\mathbf{H}(x, \mathbf{U})-\frac{\partial \mathbf{F}(x, \mathbf{U})}{\partial x}=\left(\begin{array}{c}
0 \\
g A\left(S_{0}-S_{f}-\frac{d h}{d x}+\frac{1}{B} \frac{d A}{d x}\right)
\end{array}\right)
$$

134 where the equivalence between the partial and total $x$-derivatives of the con- 
served variable $A$ should be noted. The Jacobian matrix (19) can be diago-

nalized

$$
\mathbf{J}=\mathbf{P} \Lambda \mathbf{P}^{-1}, \quad \Lambda=\mathbf{P}^{-1} \mathbf{J} \mathbf{P}
$$

${ }_{137}$ where the diagonal matrix $\boldsymbol{\Lambda}$ is formed by the eigenvalues of $\mathbf{J}$, and $\mathbf{P}$ is 138 constructed with its eigenvectors.

$$
\begin{gathered}
\mathbf{P}=\left(\begin{array}{cc}
1 & 1 \\
\lambda_{1} & \lambda_{2}
\end{array}\right), \quad \boldsymbol{\Lambda}=\left(\begin{array}{cc}
\lambda_{1} & 0 \\
0 & \lambda_{2}
\end{array}\right), \\
\mathbf{e}_{k}=\left(\begin{array}{c}
1 \\
\lambda_{k}
\end{array}\right), \quad \lambda_{1}=u-c, \quad \lambda_{2}=u+c
\end{gathered}
$$

139 The equations in non-conservative form can be discretized in a regular 140 mesh by means of the first order explicit scheme. Roe's linearization [18] 141 allows one to express the differences in the conserved variables and in the 142 source terms across the grid edge $i+1 / 2$ as a sum of waves:

$$
\begin{array}{r}
\delta \mathbf{U}_{i+1 / 2}=\mathbf{U}_{i+1}-\mathbf{U}_{i}=\sum_{m=1}^{2}\left(\tilde{\alpha}_{m} \tilde{\mathbf{e}}_{m}\right)_{i+1 / 2} \\
\left(\tilde{\mathbf{H}}^{\prime} \delta x\right)_{i+1 / 2}=\sum_{m=1}^{2}\left(\tilde{\beta}_{m} \tilde{\mathbf{e}}_{m}\right)_{i+1 / 2}
\end{array}
$$

143 with 


$$
\begin{gathered}
\tilde{\lambda}_{1}=\tilde{u}-\tilde{c}, \quad \tilde{\lambda}_{2}=\tilde{u}+\tilde{c}, \quad \tilde{\alpha}_{1}=\frac{\tilde{\lambda}_{2} \delta A-\delta Q}{2 \tilde{c}}, \quad \tilde{\alpha}_{2}=\frac{-\tilde{\lambda}_{1} \delta A+\delta Q}{2 \tilde{c}}, \\
\tilde{\beta}_{1}=-\frac{1}{2 \tilde{c}}\left\{g \tilde{A}\left[\left(\tilde{S}_{0}-\tilde{S}_{f}\right) \delta x-\delta h+\frac{1}{\tilde{B}} \delta A\right]\right\}, \quad \tilde{\beta}_{2}=-\tilde{\beta}_{1}, \\
\tilde{u}_{i+1 / 2}=\frac{\sqrt{A_{i}} u_{i}+\sqrt{A_{i+1}} u_{i+1}}{\sqrt{A_{i}}+\sqrt{A_{i+1}}}, \quad \tilde{c}_{i+1 / 2}=\sqrt{g \frac{A_{i}+A_{i+1}}{B_{i}+B_{i+1}}}
\end{gathered}
$$

$$
\left(\tilde{\nu}_{m}\right)_{i+1 / 2}=\left\{\begin{array}{lc}
\frac{1}{4}\left[\left(\lambda_{m}\right)_{i+1}-\left(\lambda_{m}\right)_{i}\right], & \text { if }\left(\lambda_{m}\right)_{i+1}>0 \text { and }\left(\lambda_{m}\right)_{i}<0 \\
0, & \text { otherwise }
\end{array}\right.
$$

146 The contributions due to the fluxes and the source terms can be expressed 147

$$
\tilde{\gamma}_{i+1 / 2}^{ \pm}=\left(\frac{1}{2}[1 \pm \operatorname{sign}(\tilde{\lambda})] \tilde{\gamma} \pm \tilde{\nu} \tilde{\alpha}\right)_{i+1 / 2}
$$

where

$$
\tilde{\gamma}_{i+1 / 2}=(\tilde{\lambda} \tilde{\alpha}-\tilde{\beta})_{i+1 / 2}
$$

149 Therefore, the first order explicit upwind numerical scheme is formulated 


$$
\Delta \mathbf{U}_{i}^{n}=-\frac{\Delta t_{1 D}}{\delta x}\left[\sum_{m}\left(\tilde{\gamma}_{m}^{+} \tilde{\mathbf{e}}_{m}\right)_{i-1 / 2}+\sum_{m}\left(\tilde{\gamma}_{m}^{-} \tilde{\mathbf{e}}_{m}\right)_{i+1 / 2}\right]^{n}
$$

It illustrates that the in-going contributions from left and right walls are used to update the value of the conserved variables at every cell (see Figure 2).

The scheme so built has been proved to be robust, conservative, well-balanced and positivity preserving [22].

The time step $\Delta t_{1 D}$ is dynamically chosen following this expression

$$
\Delta t_{1 D}=\mathrm{CFL} \min _{i, m}\left(\frac{\delta x_{i}}{\left|\tilde{\lambda}_{m}\right|_{i}^{n}}\right), \quad \mathrm{CFL} \leq 1
$$

where CFL is the Courant-Friedrich-Lewy number.

\subsection{D numerical scheme}

In the same way, it is possible to define a Jacobian matrix of the normal flux in the 2D model:

$$
\mathbf{J}=\frac{\partial(\mathbf{E} \cdot \mathbf{n})}{\partial \mathbf{U}}=\left(\begin{array}{ccc}
0 & n_{x} & n_{y} \\
c^{2} n_{x}-u \mathbf{u} \cdot \mathbf{n} & u n_{x}+\mathbf{u} \cdot \mathbf{n} & u n_{y} \\
c^{2} n_{y}-v \mathbf{u} \cdot \mathbf{n} & v n_{x} & v n_{y}+\mathbf{u} \cdot \mathbf{n}
\end{array}\right)
$$

with $\mathbf{n}=\left(n_{x}, n_{y}\right)^{T}$ the outward normal vector, $u=q_{x} / h, v=q_{y} / h, c=\sqrt{g h}$ and $\mathbf{u} \cdot \mathbf{n}=u n_{x}+v n_{y}$. Following the same philosophy, the Jacobian matrix 


$$
\begin{gathered}
\mathbf{P}=\left(\begin{array}{ccc}
1 & 0 & 1 \\
u-c n_{x} & -c n_{y} & u+c n_{x} \\
v-c n_{y} & c n_{x} & v+c n_{y}
\end{array}\right), \quad \boldsymbol{\Lambda}=\left(\begin{array}{ccc}
\lambda_{1} & 0 & 0 \\
0 & \lambda_{2} & 0 \\
0 & 0 & \lambda_{3}
\end{array}\right), \\
\mathbf{e}_{1}=\left(\begin{array}{c}
1 \\
u-c n_{x} \\
v-c n_{y}
\end{array}\right), \quad \mathbf{e}_{2}=\left(\begin{array}{c}
0 \\
-c n_{y} \\
c n_{x}
\end{array}\right), \quad \mathbf{e}_{3}=\left(\begin{array}{c}
1 \\
u+c n_{x} \\
v+c n_{y}
\end{array}\right), \\
\lambda_{1}=\mathbf{u} \cdot \mathbf{n}-c, \quad \lambda_{2}=\mathbf{u} \cdot \mathbf{n}, \quad \lambda_{3}=\mathbf{u} \cdot \mathbf{n}+c
\end{gathered}
$$

164

$$
\delta \mathbf{U}_{k}=\mathbf{U}_{j}-\mathbf{U}_{i}=\widetilde{\mathbf{P}}_{k} \tilde{\mathbf{A}}_{k}
$$

167 where $i, j$ are the indexes of the cells sharing the edge $k$ and $\tilde{\mathbf{A}}_{k}=\left(\tilde{\alpha}_{1}, \tilde{\alpha}_{2}, \tilde{\alpha}_{3}\right)_{k}^{T}$ contains the set of wave strengths. Following the linearization concept, the source term is included in the Riemann solver as a singular source. Considering that source terms are not necessarily constant in time, the following 
time linearization of the non-conservative term is applied [24]:

$$
\mathbf{H}_{k}^{n}=\left(\begin{array}{c}
0 \\
-g \tilde{h}\left(\delta z+S_{f, \mathbf{n}}\right) n_{x} \\
-g \tilde{h}\left(\delta z+S_{f, \mathbf{n}}\right) n_{y}
\end{array}\right)
$$

${ }_{172}$ The source term is next projected onto the matrix eigenvectors basis [24]

$$
\tilde{\mathbf{H}}_{k}=\widetilde{\mathbf{P}}_{k} \tilde{\mathbf{B}}
$$

173 where $\tilde{\mathbf{B}}_{k}=\left(\tilde{\beta}_{1}, \tilde{\beta}_{2}, \tilde{\beta}_{3}\right)_{k}^{T}$ contains the source strengths.

$$
\begin{gathered}
\left.\left.\tilde{\alpha}_{1}=\frac{\delta h}{2}-\frac{1}{2 \tilde{c}}(\delta \mathbf{q} \cdot \mathbf{n}-\tilde{\mathbf{u}} \cdot \mathbf{n} \delta h), \quad \tilde{\alpha}_{2}=\frac{1}{\tilde{c}}\left[\delta q_{y}-\tilde{v} \delta h\right) n_{x}-\left(\delta q_{x}-\tilde{u} \delta h\right) n_{y}\right)\right], \\
\tilde{\alpha}_{3}=\frac{\delta h}{2}+\frac{1}{2 \tilde{c}}(\delta \mathbf{q} \cdot \mathbf{n}-\tilde{\mathbf{u}} \cdot \mathbf{n} \delta h), \\
\tilde{\beta}_{1}=-\frac{1}{2 c}\left(\delta z+S_{f, \mathbf{n}}\right), \quad \tilde{\beta}_{2}=0, \quad \tilde{\beta}_{3}=-\tilde{\beta}_{1} \\
\tilde{u}_{k}=\frac{\sqrt{h_{i}} u_{i}+\sqrt{h_{j}} u_{j}}{\sqrt{h_{i}}+\sqrt{h_{j}}}, \quad \tilde{v}_{k}=\frac{\sqrt{h_{i}} v_{i}+\sqrt{h_{j}} v_{j}}{\sqrt{h_{i}}+\sqrt{h_{j}}}, \quad \tilde{c}_{k}=\sqrt{g \frac{h_{i}+h_{j}}{2}}
\end{gathered}
$$

where $\tilde{\mathbf{u}} \cdot \mathbf{n}=\tilde{u} n_{x}+\tilde{v} n_{y}, \delta \mathbf{q} \cdot \mathbf{n}=\delta q_{x} n_{x}+\delta q_{y} n_{y}$ and the averages states at each wall $k$ are represented with the tilde variables. The entropy fix for the $2 \mathrm{D}$ numerical scheme can be found in [24]. The contributions due to the fluxes and the source terms are combined in a compact expression as follows: 


$$
\tilde{\gamma}_{k}^{-}=\frac{1}{2}\left[1-\operatorname{sign}\left(\tilde{\lambda}_{k}\right)\right] \tilde{\gamma}_{k} \quad \tilde{\gamma}_{k}=(\tilde{\lambda} \tilde{\alpha}-\tilde{\beta})_{k}
$$

$$
\Delta \mathbf{U}_{i}^{n}=-\frac{\Delta t_{2 D}}{\Omega_{i}} \sum_{j=1}^{N_{E}} \sum_{m=1}^{3}\left(\tilde{\gamma}_{m}^{-} \tilde{\mathbf{e}}_{m} l\right)_{k}^{n}
$$

In this expression, $N_{E}$ indicates the number of edges in cell $i$ and $l_{k}$ is the length of each wall edge (see Figure 3). This scheme has been proved to be robust, conservative, well-balanced and positivity preserving even in presence of wet/dry fronts over irregular bed [24].

When considering unstructured meshes in the 2D scheme, the equivalent distance to $\delta x$, that will be referred to as $\chi_{i}$ in each cell, is defined by using the area of the cell as well as the length of the $k$ edges:

$$
\chi_{i}=\frac{A_{i}}{\max _{k=1, N_{E}} l_{k}}
$$

Therefore, the time step is again chosen by using the following rule:

$$
\Delta t_{2 D}=\mathrm{CFL} \frac{\min \left(\chi_{i}, \chi_{j}\right)}{\max _{m}\left|\tilde{\lambda}^{m}\right|} \quad \mathrm{CFL} \leq 1
$$




\section{Coupling strategies}

The strategies presented here to couple the $1 \mathrm{D}$ and the $2 \mathrm{D}$ models are built forcing in all cases mass conservation. For the coupled model, a new element of discretization called coupling zone is defined as the region involving the $1 \mathrm{D}$ and $2 \mathrm{D}$ cells from the discretizations of each model. It always contains one $1 \mathrm{D}$ cell and a number $N_{C}$ of adjacent $2 \mathrm{D}$ cells. Figure 4 shows two examples of coupling zones composed by only one 1D cell and some unstructured triangular 2D cells. As can be seen, two possible configurations may be defined with respect to the 1D model: frontal and lateral coupling. The discontinuous line represents the exact place where models connect between them in both configurations.

\subsection{Time step choice and model interaction}

A common element in both $1 \mathrm{D}$ and $2 \mathrm{D}$ models is the evaluation of the time step. When dynamically computed from the CFL condition, $\Delta t$ can be different in both models. The global $\Delta t$ taken is the minimum value of the two models, that is:

$$
\Delta t=\min \left(\Delta t_{1 D}, \Delta t_{2 D}\right)
$$

Once $\Delta t$ is calculated, each model computes separately its own conserved variables according to (30) and (39). The resulting values, not including yet the interaction between the two models will be called from now on star variables. So, in each coupling zone, $(A, Q)^{*}$ and $(h, h u, h v)^{*}$ are provided 
by the $1 \mathrm{D}$ and the $2 \mathrm{D}$ model respectively. Then, mass and/or momentum conservation is enforced so that the variables can be updated and finally $\Delta t$ is increased. The flowchart of the coupled model is summarized in Figure 5. Two different coupling strategies are presented on the basis that the computational domains connect at each coupling zone and the boundary conditions and their treatment must be continuously revisited. This is a strong point that justifies the novelty of this work in terms of mass and momentum conservation and that will be also important when choosing the adequate coupling strategy providing the flow regime at the boundary.

\subsection{Only Mass Conservation (OMC)}

This technique consists of imposing the same water level in the coupling zone considering the involved cells (1D and 2D computational cells) as a single domain where the water volume conservation is enforced. The common water level is based on a strict mass conservation. Both models are coupled by considering the information that crosses the internal boundaries of the coupling zone as relevant in terms of mass conservation [25]. Figure 6 illustrates the contributions to be considered in a frontal coupling and in a pure lateral coupling.

The total water volume of a coupling zone, $V_{C Z}$ can be written as follows:

$$
V_{C Z}=A_{1 D}^{*} \delta x+\sum_{i}^{N_{C}} h_{i}^{*} S_{i}+Q_{1 D}^{n} n_{1 D} \Delta t+\sum_{i}^{N_{C}}\left(\mathbf{F}_{1 i}^{n} \cdot \mathbf{n}_{i} l_{i}\right) \Delta t
$$


where

$$
A_{1 D}^{n+1}=A_{1 D}^{n+1}\left(z_{s}^{n+1}\right) \quad h_{i}^{n+1}=z_{s}^{n+1}-z_{b_{i}}
$$
the water volume in the $N_{C} 2 \mathrm{D}$ cells ( $S_{i}$ is the $2 \mathrm{D}$ cell size), $Q_{1 D}^{n} n_{1 D} \Delta t$ represents the volume due to the 1D-flow that crosses the 'discontinuous line' separating the two models and $\sum_{i}^{N_{C}}\left(\mathbf{F}_{1 i}^{n} \cdot \mathbf{n}_{i} l_{i}\right) \Delta t$ gives information about the water volume provided by the 2D-flow going across the boundaries, where $\mathbf{F}_{1 i}^{n}=\left(q_{x}, q_{y}\right), \mathbf{n}_{i}$ the outward normal direction and $l_{i}$ the length of each wall taking part in the coupling zone. It is easy to see that $n_{1 D}= \pm 1$ in the frontal coupling and $n_{1 D}=0$ in pure lateral coupling.

Once $V_{C Z}$ is computed, a new common water level surface $z_{s}^{n+1}$ is imposed in the coupling zone by distributing correctly the water volume in the $1 \mathrm{D}$ and the $2 \mathrm{D}$ system:

$$
V_{C Z}=A_{1 D}^{n+1} \delta x+\sum_{i}^{N_{C}} h_{i}^{n+1} S_{i}
$$

The calculation of the new water surface level $z_{s}^{n+1}$ is not trivial when dealing with complex topography and is explained in Appendix A.

\subsection{Mass and Momentum Conservation (MMC)}

The aim consists of the extrapolation of the idea used in the $O M C$ strategy in order to achieve, apart from the mass conservation, also the exact

where $A_{1 D}^{*} \delta x$ is the volume of water in the $1 \mathrm{D}$ cell, $\sum_{i}^{N_{C}} h_{i}^{*} S_{i}$ accounts for 


$$
M_{x}=Q_{x 1 D}^{*} \delta x+\sum_{i}^{N_{C}}\left(q_{x}\right)_{i}^{*} S_{i}+E_{x}^{n} n_{1 D} \Delta t+\sum_{i}^{N_{C}}\left(\mathbf{F}_{2 i}^{n} \cdot \mathbf{n}_{i} l_{i}\right) \Delta t
$$

${ }_{254}$ where $Q_{x 1 D}^{*} \delta x$ is the momentum in the $1 \mathrm{D}$-system, $\sum_{i}^{N_{C}}\left(q_{x}\right)_{i}^{*} S_{i}$ in the 2D-

global momentum conservation. For this purpose, not only a common water level surface is imposed at the coupling zone but also the velocities in $x$ and $y$-direction are both shared.

In the $M M C$ strategy, the information about the flow direction is of interest, hence an angle $\theta$ is introduced in the $1 \mathrm{D}$ model in order to express the discharge $Q_{1 D}$ as a vector:

$$
Q_{1 D} \rightsquigarrow\left(Q_{x 1 D}, Q_{y_{1 D}}\right)=\left(Q_{1 D} \cos \theta, Q_{1 D} \sin \theta\right)
$$

Using the same procedure as in $O M C$ and also involving the same information, it is possible to define the amount of momentum in $x$-direction, $M_{x}$, as system and $E_{x}^{n}$ and $\mathbf{F}_{2 i}^{n}$ accounts for the corresponding fluxes that cross the boundary shared by 1D and 2D models:

$$
E_{x}^{n}=\left(\frac{\left(Q_{x}\right)^{2}}{A}+g I_{1}\right)_{1 D}^{n} \quad \mathbf{F}_{2 i}^{n}=\left(\frac{q_{x}^{2}}{h}+\frac{1}{2} g h^{2}, \frac{q_{x} q_{y}}{h}\right)_{i}^{n}
$$

The momentum considering the $y$-direction: 


$$
M_{y}=Q_{y_{1 D}^{*}}^{*} \delta x+\sum_{i}^{N_{C}}\left(q_{x}\right)_{i}^{*} S_{i}+E_{y}^{n} n_{1 D} \Delta t+\sum_{i}^{N_{C}}\left(\mathbf{F}_{3 i}^{n} \cdot \mathbf{n}_{i} l_{i}\right) \Delta t
$$

where

$$
E_{y}^{n}=\left(\frac{\left(Q_{y}\right)^{2}}{A}+g I_{1}\right)_{1 D}^{n} \quad \mathbf{F}_{3 i}^{n}=\left(\frac{q_{x} q_{y}}{h}, \frac{q_{y}^{2}}{h}+\frac{1}{2} g h^{2}\right)_{i}^{n}
$$

259 It is clear again that $n_{1 D}= \pm 1$ in the frontal coupling and $n_{1 D}$ becomes nil in pure lateral coupling.

261

Once $M_{x}$ and $M_{y}$ are computed, common average velocity components in $x$-direction, $\bar{u}$, and in $y$-direction, $\bar{v}$, can be derived from the total water volume in the coupling zone $V_{C Z}$ using

$$
V_{C Z} \bar{u}=M_{x} \quad V_{C Z} \bar{v}=M_{y}
$$

264 Finally, the conserved variables are updated as:

$$
\begin{gathered}
\left(q_{x}\right)_{i}^{n+1}=h_{i}^{n+1} \bar{u} \quad\left(q_{y}\right)_{i}^{n+1}=h_{i}^{n+1} \bar{v} \\
Q_{1 D}^{n+1}=A_{1 D}^{n+1}(\bar{u} \cos \theta+\bar{v} \sin \theta)
\end{gathered}
$$




\subsection{Boundary conditions and the choice of the adequate coupling strategy}

When detailing the coupling strategies presented before $(O M C, M M C)$, the information that crosses the internal line that links both models has been included in order to contemplate a fully conservative method. However, it is not the only point to be considered. For example, in a frontal coupling configuration, where the end of the 1D domain connects with the beginning of the 2D system, the boundary conditions are entirely present and it is relevant whether a supercritical or subcritical flow is present at the coupling zone. Therefore, the boundary condition treatment must be analysed in order to achieve a complete 1D-2D coupling model.

In the case of a hyperbolic system, the theory of characteristics provides clear information about the number of external boundary conditions to be imposed at the inlet or at the outlet domain [26]. The set of possibilities is illustrated for the 1D case in Figure 7 and can be summarized as follows $[25,27]$ :

1. Subcritical inlet flow: One of the variables is enforced and the other are calculated numerically.

2. Supercritical inlet flow: All the variables have to be imposed, no information is provided from the inner cells.

3. Subcritical outlet flow: As in the subcritical inlet flow, one of the variables is required to complete the information at the boundary cell.

4. Supercritical outlet flow: No extra information apart from that computed numerically is needed. 
This information must be carefully handled for building the coupled model. In order to distinguish the flow regime, the Froude number is evaluated separately in both models, at each coupling zone. When either the 1D or the $2 \mathrm{D}$ model contains a supercritical boundary, the $M M C$ strategy, in-

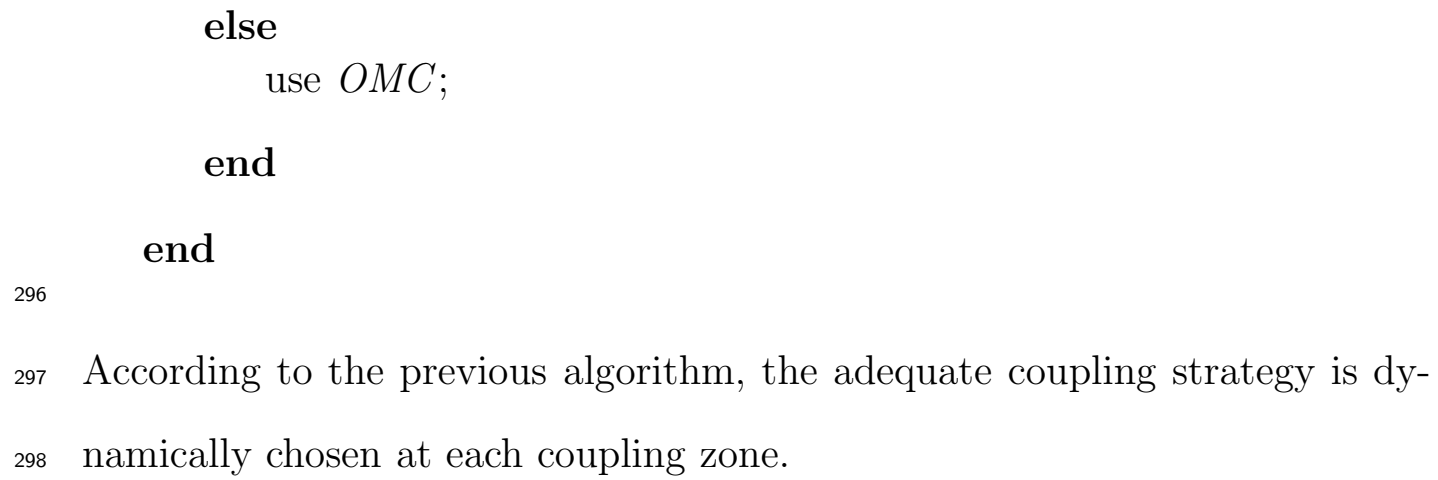

According to the previous algorithm, the adequate coupling strategy is dynamically chosen at each coupling zone. 


\section{Numerical results}

\subsection{Test case 1: Dam-break in a channel with a flood plain}

In this section, the simulation of a dam break in a channel that ends into a flood plain [19] is presented. The test case was designed and measured in the National Laboratory of Civil Engineering in the IST in Portugal. Initial conditions are $\mathrm{h}=0.504 \mathrm{~m}$ at the reservoir and $\mathrm{h}=0.003 \mathrm{~m}$ in the rest of the channel and flood plain [28]. Solid walls are all around except at the outlet that is free (see Figure 8). There is no elevation in the domain and the friction was previously calibrated with a Manning coefficient of $n=0.009 \mathrm{~s} / \mathrm{m}^{\frac{1}{3}}$. The time evolution of the water depth was measured at P1, P2, P3, P4, P5 and P6 displayed in Figure 8.

Figure 9 illustrates the discretization used for both frontal and lateral configurations where the mesh used in the $2 \mathrm{D}$ domain of the coupled model is unstructured triangular grid. Apart from experimental data, the fully 2D model, used as reference solution whose discretization is composed by 8760 unstructured triangular cells and the fully 1D model, discretized with $\delta x=0.1$ $m$ are also included in order to evaluate the relative behaviour of the proposed coupled model. It is worth emphasizing here that the main objective of this work is to evaluate whether the coupled model is able to produce numerical results at least equal to the those from the $2 \mathrm{D}$ numerical model but at a reduced computational cost.

Despite the apparent simplicity, this test case represents a dambreak flow with large Froude numbers (near to 4) at the location of the wave front. 
The measurements of the water depth contain an experimental uncertainty. As the water depth values are relatively small in this test case (around centimetres) the experimental error is rather noticeable in some probes showing oscillatory experimental data. As already noticed in [28], the numerical models are sometimes unable to reproduce exactly these observances.

Figure 10 shows the comparison of the numerical results obtained with the $1 \mathrm{D}-2 \mathrm{D}$ frontal and lateral coupling, the fully $1 \mathrm{D}$ model, the fully $2 \mathrm{D}$ model and the experimental measurements in time evolution of the depth of water at the gauge points. Attending to probes P2 and P6, both the 1D2D frontal and lateral coupling models are able to reproduce faithfully the experimental measurements being very similar to the fully $2 \mathrm{D}$ approach. At probe P1, located within the narrow region, the predictions of all models almost coalesce but they are all unable to approximate well the experimental data. This was already noticed in the original work [28]. The same happens at probe $\mathrm{P} 4$, where unexpected oscillatory measurements are not reproduced by any model. The behaviour at probe P3 is slightly different. The 1D2D lateral coupling model does not approximate accurately the experimental data at this probe due to the $1 \mathrm{D}$ cross section averaging and the $1 \mathrm{D}-2 \mathrm{D}$ frontal coupling strategy propagates the flood wave slower than the fully $2 \mathrm{D}$ model or the experimental data. Probe 5 is located near the lateral wall were the shock wave reflects so that it shows first the arrival of the front and then the arrival of the reflected wave. The fully $2 \mathrm{D}$ model and the $1 \mathrm{D}-2 \mathrm{D}$ frontal coupled model, with all the floodplain considered as a 2D domain, show the 
best results. They compare to those reported in [28]. The 1D-2D lateral coupled model performs worse due to the forced average values introduced along the 1D domain.

On the other hand, as expected, the fully 1D model, which represents the floodplain as a sudden enlargement, propagates a flood wave faster than the $2 \mathrm{D}$ model, giving unrealistic results and providing the worst numerical approximation.

The coupling model designed is able to detect dynamically the Froude numbers at each side of the coupling zone and determine the adequate technique at each moment. For example, the nature of this test case indicates that, during the simulation time, the coupling technique internally adopted by the model is always the $M M C$ strategy due to the supercritical flow regime.

It is worth emphasizing that boundary conditions play an important role in the coupling model proposed in this work. Therefore, a remarkable statement derived from the information needed at the boundaries is that not any strategy is valid for computing certain scenarios. For this purpose, the same dambreak test case is simulated enforcing a $O M C$ strategy everywhere all the time. Results are shown in Figure 11, plotting the experimental measurements at the known gauge points against the $O M C$ approach results. The $O M C$ strategy is unable to approximate the experimental data at almost any of the gauge points, providing also non-physical results as a consequence of a wrong boundary treatment at the coupling zone where less information than 
the necessary is provided.

\subsection{Test case 2: Channel with a lateral floodplain area}

This academic test case deals with a $2000 \mathrm{~m}$ long and $68 \mathrm{~m}$ base wide trapezoidal channel connected laterally with a floodplain area (Figure 12). A slope of $1 / 1000$ is assumed and the friction is modelled by using different Manning coefficients: $\mathrm{n}=0.015 \mathrm{~s} / \mathrm{m}^{\frac{1}{3}}$ in the river bed and $\mathrm{n}=0.03 \mathrm{~s} / \mathrm{m}^{\frac{1}{3}}$ in the lateral floodplain.

Being a synthetic test case, the numerical results obtained by the coupled model will be compared with a fully $2 \mathrm{D}$ model through 10 probes situated in the floodplain area. A sketch of the test case containing the location of the probes can be observed in Figure 13.

The comparison with a fully $2 \mathrm{D}$ model is only a good measure of the behaviour of a new coupled model when the mesh is fine enough. Unless the previous test case, the channel and floodplain are not flat. The 2D grid refinement should follow the necessity to represent faithfully the topography. In this case, the topography is represented by the $2 \mathrm{D}$ model through computational cells covering all the domain and the representation of the terrain is as accurate as the mesh resolution. The trapezoidal cross section is represented by unstructured triangular cells (Figure 14) so that, if a fine discretization is not applied, some errors can be derived from this aspect.

Another source of uncertainty is the meaning and the interpretation of the Manning friction coefficient in each model and the relative connection or 
correspondence between the $1 \mathrm{D}$ or the $2 \mathrm{D}$ model $[29,30]$. This topic is out of the scope of this work. However, it must be considered when the numerical results achieved by a 1D-2D coupled model would like to be compared with a fully $2 \mathrm{D}$ model and to determine what is the error associate to this parameter.

Two scenarios involving lateral coupling are simulated in order to evaluate again the performance of the scheme: steady and unsteady flow.

\subsubsection{Steady flow}

A constant discharge of $600 \mathrm{~m}^{3} / \mathrm{s}$ is introduced as the upstream inlet boundary condition and the model is run until convergence to steady state. A gauging curve is used as outlet boundary condition at the end of the channel. The numerical results obtained by the coupled model are compared with a fully 2D model in terms of longitudinal profile along the channel center line once the steady state is reached (Figure 15) and also registering the time evolution of the water depth at each probe (Figures 16 and 17).

Observing the results, an almost constant difference is appreciated between the fully 2D numerical model and the proposed 1D-2D coupling model. As the difference is almost constant in all the probes it may indicate that the deviation is due to the Manning roughness coefficient in the river bed and its adjustment for each model. In both simulations, the choice of this coefficient has the same value but, however, it is underestimated by the 1D approach (included in the coupled model) achieving a lower water depth in the time evolution of each probe. 
In order to corroborate this hypothesis, a new simulation has been carried out by tuning manually the Manning coefficient to $\mathrm{n}=0.01605 \mathrm{~s} / \mathrm{m}^{\frac{1}{3}}$ in the river bed for the coupled model. The results can be observed in Figure 18 plotting the longitudinal profile all along the channel and in Figures 19 and 20, where the water depth time evolution is registered for both models.

As shown, the results obtained by the coupled model coalesce almost exactly with the same obtained with the fully $2 \mathrm{D}$ model. In particular, the behaviour at probe 6 that is always 'dry' is emulated in both models.

The Froude number is less than one in all the domain, so the test case is always developed in a subcritical regime. Therefore, the coupled model is automatically using the $O M C$ strategy during all the simulation in order to compute the water surface level at each coupling zone. In order to check again the importance of the boundary treatment, the same case is simulated enforcing the $M M C$ strategy. Results are shown in terms also of longitudinal profile and time evolution of water depth at each measurement point in Figures 21, 22 and 23. When using the $M M C$ strategy in presence of a subcritical regime, more information than necessary is provided, and the numerical solution achieved by the coupled model is far from that obtained by the fully $2 \mathrm{D}$ model arriving to non-physical results.

\subsubsection{Unsteady flow}

Adopting the modified Manning roughness coefficient $\mathrm{n}=0.01605 \mathrm{~s} / \mathrm{m}^{\frac{1}{3}}$ in the river bed for the coupled model, a new comparison is proposed by 
using the same test case now considering unsteady flow. A triangular inlet discharge hydrograph (Figure 24) with a peak discharge of $600 \mathrm{~m}^{3} / \mathrm{s}$ is introduced to the system. The water depth time evolution at the gauge points (Figure 13) resulting from the coupled model is compared again with a fully 2D model in Figures 25 and 26.

A good agreement is achieved between both sets of numerical results. Not only the shape of the probes registering some water is respected but also the absence of water at probes 1,6 and 7 is well reproduced by the coupled model.

\subsection{Test case 3: Convergence to steady state in a $Y$ junction}

Two cases of numerical simulation of the evolution of flow towards steady state at a junction of three channels of large slope are next presented. The interest of this test case lies in the changing flow regime due to the configuration of the system hence the dynamically choice of the adequate strategy $(O M C$ or $M M C)$.

A rectangular cross section channel $1 \mathrm{~m}$ wide (channel 1) branching into two channels of the same geometry (channels 2 and 3) are considered. A constant discharge of $3 \mathrm{~m}^{3} / \mathrm{s}$ is assumed at the inlet point to channel 1 and a fixed Froude number of 0.14 is enforced at the outlet of channels 2 and 3, starting from the initial conditions of uniform water depth of $2 \mathrm{~m}$.

As experimental data are not available for this test case, a fully $2 \mathrm{D}$ model is used to compare with the results achieved by the proposed $1 \mathrm{D}-2 \mathrm{D}$ cou- 
pled model. The comparison will be made through the longitudinal profiles achieved by each model as well as through several probes or gauge points placed all along the domain, including the three channels and the junction location. The exact position of the probes is shown in Figure 27.

Two configurations are proposed by changing the bed slope of each channel, leading to different flow regimes. The Manning roughness coefficient is uniformly chosen as $n=0.009 \mathrm{~s} / \mathrm{m}^{\frac{1}{3}}$.

\subsubsection{Supercritical junction}

In this example, the values of bed slope

$$
S_{0_{1}}=S_{0_{2}}=S_{0_{3}}=0.01
$$

The steady state is reached starting from a fully subcritical flow due to the initial condition. When convergence to steady state is achieved, the flow is supercritical all over the domain except for the downstream part of channels 2 and 3 , in which identical hydraulic jumps develop to connect with the outlet boundary condition at these locations (Figure 28). The results in terms of water level surface at each probe are plotted in Figure 29 where the coupled model is represented in shadows symbols and the fully $2 \mathrm{D}$ model in empty symbols as before.

As can be observed, there is a good agreement between both numerical approaches in all the probes. 


\subsection{Subcritical junction}

With another choice of the bed slope,

$$
S_{0_{1}}=0.01 \quad S_{0_{2}}=S_{0_{3}}=0.001
$$

the equilibrium flow reached is subcritical at the junction but discontinuous in channel 1, where a hydraulic jump connects the two regimes. Therefore, channels 2 and 3 remain always in a subcritical regime. The longitudinal profile for this configuration, when the steady state is reached, is plotted in Figure 30. Also numerical results concerning the evolution in time of the water level surface from the coupled model and the fully 2D model are shown in Figure 31.

The results are almost the same in the fully $2 \mathrm{D}$ model and in the coupled model. In particular some oscillations appear in probes 3 to 8 due to the proximity of the hydraulic jump which are well reproduced by the coupled model.

\subsection{Test case 4: Real world configuration in a meandering river reach}

A case study based on a reach of the Ebro river near urban area (see Figure 32) has been selected to evaluate the uncertainty in the flooding predictions introduced by the choice of the proposed coupled model. The Digital Terrain Model (DTM) used in this work was provided by the Ebro River Basin Administration (www.chebro.es). It had been obtained using the Laser Induced Direction And Ranging (LIDAR) data, by means of a test programme 
using a single pulse scanning sensor, with $0.10 \mathrm{~m}$ vertical accuracy and $1 \mathrm{~m}$ horizontal resolution. The DTM provides data of great accuracy, but does not furnish any information of the region covered by the water. However, the uncertainty on the particular shape of the river bed under the water surface has been eliminated by reconstructing the river able to convey the water discharge that was flowing in the moment of the LIDAR measurements and so that it reproduces the water surface extension and slope as measured. The DTM plus the river bed reconstruction were used as a full bed topography to provide information to both 2D and coupled models.

Two scenarios have been carried out in order to see the performance of the coupled scheme: steady and unsteady flow. Not having an exact solution or measured data in this river reach, the numerical solution from a fully tested 2D simulation model with a fine grid of 200000 unstructured triangular cells (Figure 33 (left)) has been used as a reference solution. In the coupled model, the floodplain inundation is clearly complex hence requiring a $2 \mathrm{D}$ model when numerical simulation is sought as more than one flow direction are relevant. Therefore, the river bed will be simulated with a 1D model laterally connected with the $2 \mathrm{D}$ model. A detail of the coupling model domains is shown in Figure 33 (right). The discretization in the 1D model is made of 112 cross sections and the 2D domain is covered by almost 46000 triangular cells.

The 2D computations use a single Manning coefficient $\mathrm{n}=0.035 \mathrm{~s} / \mathrm{m}^{\frac{1}{3}}$ all over the domain. However, the 1D scheme 'inside' the coupled model needs 
a greater coefficient in order to diminish the differences with a 2D model. For this purpose, $\mathrm{n}=0.035 \mathrm{~s} / \mathrm{m}^{\frac{1}{3}}$ has been chosen all along the $2 \mathrm{D}$ domain and $\mathrm{n}=0.038 \mathrm{~s} / \mathrm{m}^{\frac{1}{3}}$ in the $1 \mathrm{D}$ sub-domain of the coupled model.

\subsection{Steady flow}

The generation of steady state conditions in the river reach has been achieved by convergence to the steady state starting from an empty or dry river. It consists of applying a constant upstream discharge of $600 \mathrm{~m}^{3} / \mathrm{s}$ until the river reach fills up and the outlet discharge is equal to the inlet discharge. Figure 34 illustrates the flooding map predicted by the 2D model (left) and by the $1 \mathrm{D}-2 \mathrm{D}$ coupled model (right). In the $1 \mathrm{D}-2 \mathrm{D}$ representation, the $1 \mathrm{D}$ sub-domain shows the cross sectional basis whereas the $2 \mathrm{D}$ sub-domain is meshed in triangles. A zoom view of the flooding area has been highlighted. The coupled model approaches very finely the results predicted by the fully 2D model respecting the wet and the dry regions. Moreover, having a coarse representation of the information in the 1D domain (only 112 sections), the color scale for the river bed elevation is almost exactly reproduced. In order to corroborate this hypothesis, the longitudinal profiles along the river centerline, achieved by each numerical model is plotted in Figure 35. As can be appreciated, there is a good agreement between the 2D numerical model and the coupled scheme.

The flooded area predicted by each model represents another measurement of the quality of the results. Table 1 contains the information about 
the flooded area computed by the 1D-2D coupled model as well as by the $2 \mathrm{D}$ model. The relative error (less than 3\%) shows that the proposed coupled scheme is able to approximate well the results achieved by the $2 \mathrm{D}$ model.

\subsection{Unsteady flow}

From a $75 \mathrm{~m}^{3} / \mathrm{s}$ steady state, unsteady calculations were performed by assuming a triangular shape inlet discharge hydrograph rising to $1300 \mathrm{~m}^{3} / \mathrm{s}$ in 12 hours. The predicted flood inundations at $\mathrm{t}=50000 \mathrm{~s}$ are shown in Figure 36, computed with a fully 2D model (left) and also calculated with the proposed 1D-2D coupled model (right).

The numerical results indicate that there is a good adjustment between the fully 2D model and the coupled model, respecting mainly the wet and dry zones. Small differences are observed in the first part of the river reach, upstream the island, where the flooded zone predicted by the fully $2 \mathrm{D}$ model is larger than that provided by the coupled model. The overall color scale used shows a good agreement not only along the river bed but also over the floodplain. The longitudinal profile (see Figure 37) as well as the flooded area predicted by each model (Table 1) display the quality of the results obtained by the coupled model in comparison with the complete $2 \mathrm{D}$ model. The coupled model is able to reproduce all kind of flow situations and predict faithfully the water level surface as presented before hence it may be a $2 \mathrm{D}$ model overestimation of the flooding due to the spatial discretization of the river bed bathymetry. 


\section{Computational time}

As displayed in the previous test cases, accuracy is ensured by using the proposed coupled model. As seen, it is able to approach satisfactorily the results offered by the pure 2D model. However, the 2D model has a clear disadvantage associated to the topography discretization due to the fact that the computational time is governed by the cell sizes. Therefore, a compromise between the CPU time and the topography representation must be achieved. In particular, when dealing with a flood scenario, a wrong representation of the bottom bathymetry in the river bed entails wrong results concerning the extension of the flooding. Therefore, a fine discretization should be considered in order to ensure reasonable results with the extra cost in terms of CPU time.

The coupled model eliminates this fine discretization associate to the river bed topography since the $1 \mathrm{D}$ model is able to reproduce it very accurate, requiring less information and saving computationally time. Not only a lot of cells which discretized the river bed are discarded for the 2D domain, but also they are possibly the cells which limited the time step size. Therefore, the computational time should be reduced 'a priori' when dealing with a 1D-2D coupled model.

In order to compare the CPU time consumed, Table 2 is attached where each test case enclosed its computational time is analysed for the simulations computed by the fully $2 \mathrm{D}$ model and also by the coupled model presented before. The maximum triangle cell area constraint in the fully $2 \mathrm{D}$ model 
has been chosen equal from that of the 2D domain of the coupled model for each test case hence the uncertainty related with the choice of different computational cell sizes is eliminated.

The results highlight a computational gain achieved by using the proposed coupled model. Test case 1 is the less representative in terms of speed-up than others due to the simulation time (only 10 seconds) as well as the number of cells in the fully 2D model in comparison with the coupled model. However, when dealing with test cases 2,3 and 4 , the speed-up reached by the coupled model is not inconsiderable at all. Furthermore in a real configuration, where the $1 \mathrm{D}$ model represents only the river bed and the adjacent low-laying areas are covered by a $2 \mathrm{D}$ discretization, the gain observed is particularly noticeable, always achieved without essentially loss of accuracy.

\section{Conclusions}

A 1D-2D numerical coupled model built from existing both $1 \mathrm{D}$ and 2D models is presented in this work. The implementation of a complete 1D-2D model seems a good solution to eliminate not only the limitations of the $1 \mathrm{D}$ model related with the underlying mathematical hypothesis which introduce some errors when modelling flooding waves over 2D domains, but also the uncertainty in the $2 \mathrm{D}$ model associate to the discrete representation of the topography.

Two possible coupling techniques are displayed. The $O M C$ technique is derived from a total mass conservation in the coupling zone. A new com- 
mon level surface is established from the total water volume existing at the coupling zone. The $M M C$ strategy, considered as an extension of the $O M C$ enforces not only a new common level at the coupling zone, but also the velocities $u$ and $v$ in $x$ and $y$ direction coming from a strictly mass and momentum control. It is important to remark the importance of computing the exact mass and/or momentum conservation, considering the information that crosses the limits of the $1 \mathrm{D}$ or $2 \mathrm{D}$ domains.

The use of each strategy is not transparent to the boundary conditions of the $1 \mathrm{D}$ and the $2 \mathrm{D}$ models. The boundary treatment must be revisited and, according to the flow regime, the $O M C$ or $M M C$ strategy must be used to avoid non-physical results.

The effectiveness of the coupling model is tested trough diverse test cases where the performed numerical results of the coupling model are compared with a fully $2 \mathrm{D}$ model as well as with experimental data if existing. It has also been evaluated in a real world configuration, simulating a reach of the Ebro river by means of a 1D model connected with the riverside floodplain areas which are covered by a $2 \mathrm{D}$ domain.

Finally, the computational gain achieved by this proposed coupled model is highlighted in comparison with the CPU time consumed by a fully $2 \mathrm{D}$ model. 
[1] H. Yoshida and A. Dittrich, 1D unsteady-state flow simulation of a section of the upper Rhine, Journal of Hydrology 269 79-88 (2002).

[2] T. Helmiö, Unsteady 1D flow model of compound channel with vegetated floodplains, Journal of Hydrology 269 89-99 (2002)

[3] M. Masood and K. Takeuchi, Assessment of flood hazard, vulnerability and risk of mid-eastern Dhaka using DEM and 1D hydrodynamic model, Nat Hazards 61 757-770 (2012)

[4] A. Castellarin, A. Domeneghetti and A. Brath, Identifying robust largescale flood risk mitigation strategies: A quasi-2D hydraulic model as a tool for the Po river, Physics and Chemistry of the Earth 36 299-308 $(2011)$

[5] K-E. Lindenschmidt S. Huang and M. Baborowski, A quasi-2D flood modeling approach to simulate substance transport in polder systems for environment flood risk assessment, Science of the total environment 397 86-102 (2008)

[6] D. Meire, L. De Doncker, F. Declercq, K. Buis, P. Troch and R. Verhoeven, Modelling river-floodplain interaction during flood propagation, Nat. Hazards 55 111-121 (2010)

[7] A. Verwey, Latest development in floodplain modelling-1D/2D integration, Proceedings of the Australian Conference on Hydraulics in Civil Engineering, The Institute of Engineers, Hobart (2001) 
[8] J. F. Dhondia and G. S. Stelling, Application of one dimensional-two dimensional integrated hydraulic model for flood simulation and damage. Proceedings of the 5th International Conference on Hydroinformatics, Cardiff, pp.265-276. (2002)

[9] I.Yu. Gejadze and J. Monnier, On a 2D 'zoom' for the 1D shallow water model: Coupling and data assimilation, Comput. Methods Appl. Mech. Engrg. 196 4628-4643 (2007)

[10] J. Marin and J. Monnier, Superposition of local zoom models and simultaneous calibration for 1D-2D shallow water flows, Mathematics and Computers in Simulation 80, 547-560 (2009)

[11] B. Lin, J.M. Wicks, R.A. Falconer and K. Adams, Integrating 1D and 2D hydrodynamic models for flood simulation, Proceedings of the Institution of Civil Engineers, Water Management, 159 19-25. (2006)

[12] I. Villanueva and N.G. Wright, Linking Riemann and storage cell models for flood prediction, Water Management 159 Issue WM1 (2005)

[13] E. Miglio, S. Perotto and F. Saleri, Model coupling techniques for free surface flow problems: Part I, Nonlynear analysis 63 1885-1896. (2005)

[14] W. F. Li, Q W Chen and J Q Mao, Development of 1D and 2D coupled model to simulate urban inundation: An application to Beijing Olympic Village, Chinese Sci Bull, 2009, 54(9): 1613-1621 
[15] E. Bladé, M. Gómez-Valentin, J. Dolz, J.L. Aragón-Hernández, G. Corestein and M. Sánchez-Juny, Integration of 1D and 2D finite volume schemes for computations of water flow in natural channels, Advances in Water Resources 42 17-29 (2012)

[16] P. Finaud-Guyot, C. Delenne, V. Guinot and C. Llovel, 1D-2D coupling for river flow modeling, C. R. Mecanique 339 226-234 (2011)

[17] E.D. Fernandez-Nieto, J. Marin and J. Monnier, Coupling superposed 1D and 2D shallow-water models: Source terms in finite volume schemes, Computers and Fluids 39 1070-1082 (2010)

[18] P.L. Roe, Approximate Riemann solvers, parameter vectors and difference schemes, Journal of Computational Physics, 43, 357-372. (1981)

[19] A.B. Franco, and A.B. Almeida, Numerical and computational results of the 2D BIPLAN model. Proc. of the IV meeting on dam break modelling, Wallingford, UK. (1998)

[20] J. Burguete and P. García-Navarro, Efficient construction of highresolution TVD conservative schemes for equations with source terms: application to shallow water flows, International Journal for Numerical Methods in Fluids, 37, 209-248. (2001)

[21] J.A. Cunge, F.M. Holly, A. Verwey, Practical aspects of computational river hydraulics. Pitman Pub Inc. (1989) 
[22] J. Burguete and P. García-Navarro, Improving simple explicit methods for unsteady open channel and river flow International Journal for $\mathrm{Nu}$ merical Methods in Fluids, 45, 125-156. (2004)

[23] M. Morales-Hernández, P. García-Navarro and J. Murillo, A large time step 1D upwind explicit scheme $(\mathrm{CFL}>1)$ : application to shallow water equations, Journal of Computational Physics 231 , pp. 6532-6557 (2012)

[24] J. Murillo and P. García-Navarro, Weak solutions for partial differential equations with source terms: Application to the shallow water equations, J. Comput. Phys. 229 4327-4368. (2010)

[25] J. Burguete, P. García-Navarro and J. Murillo, Numerical boundary conditions for globally mass conservative methods to solve the shallowwater equations and applied to river flow, Int. J. for Num. Meth. In Fluids, 51 585-615 (2006)

[26] H.O Kreiss, Initial boundary value problem for hyperbolic systems. Communication of Pure and Applied Mathematics 23, 277 - 298. (1970)

[27] P. Brufau, M.E. Vázquez Cendón and P. García-Navarro, A numerical model for the flooding and drying of irregular domains, Int. J. for Num. Meth. In Fluids, 39 247-275. (2002)

[28] CADAM, 2000, CADAM, Concerted Action on Dam Break Modelling, Short history, objectives, Project report, modelling guidelines, partici- 
pants, meetings, test cases, proceedings, author index, CD Rom, Université catholique de Louvain.

[29] D. Lilas, S. Proust, A. Paquier, Analyse de la pertinence du calage du coefficient de Manning pour des crues faiblement debordantes. Proceedings Simhydro2010 Sophia-Antipolis, France (2010)

[30] H.P. Morvan, D.K. Knight, N.G. Wright, X.Tang and A.J. Crossley , The concept of roughness in fluvial hydraulics and its formulation in 1D, 2D and 3D numerical simulation models, Journal of Hydraulic Research, 46(2), 191-208. (2008) 


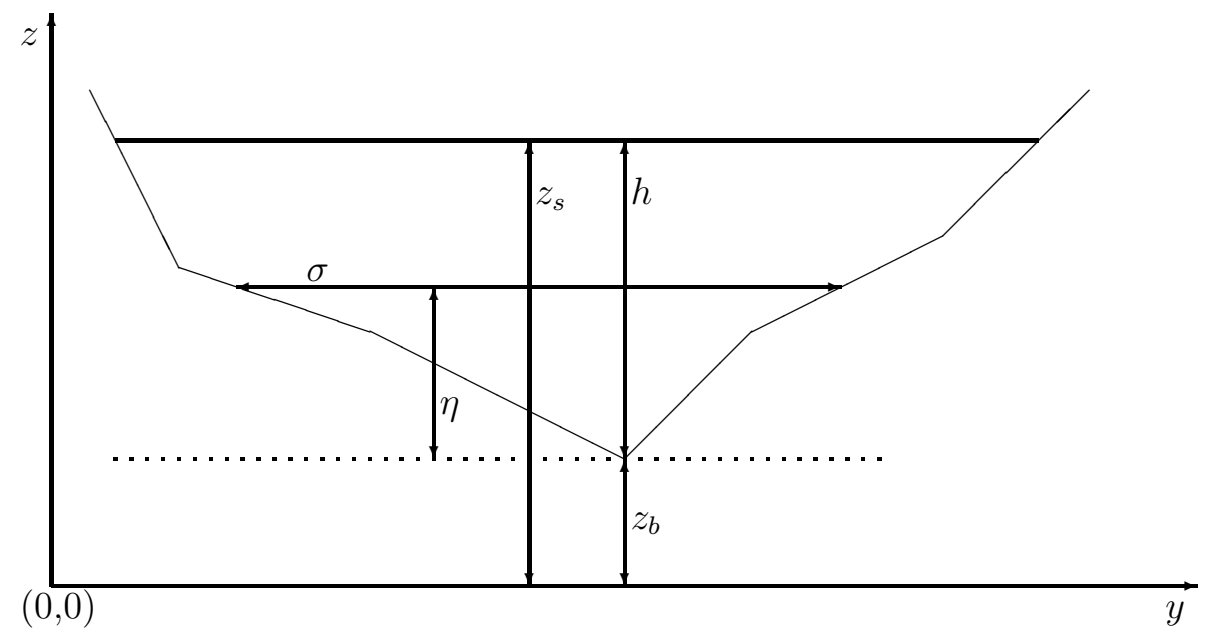

Figure 1: Coordinate system in a cross section as used in the 1D model

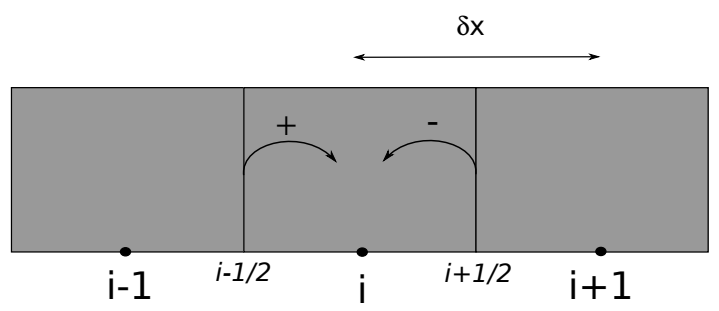

Figure 2: Scketch of the 1D numerical scheme

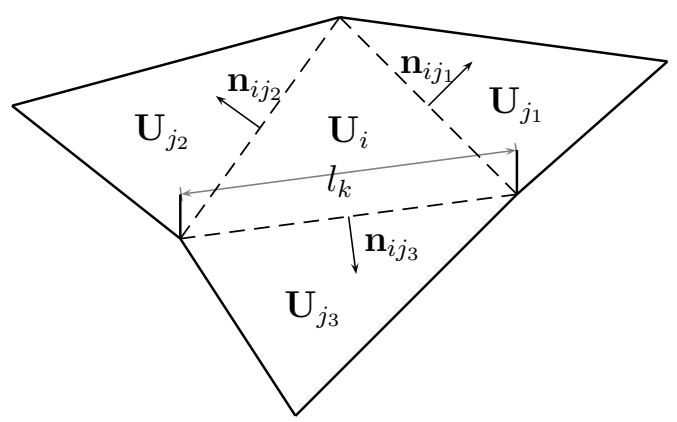

Figure 3: Sketch of the 2D numerical discretization 


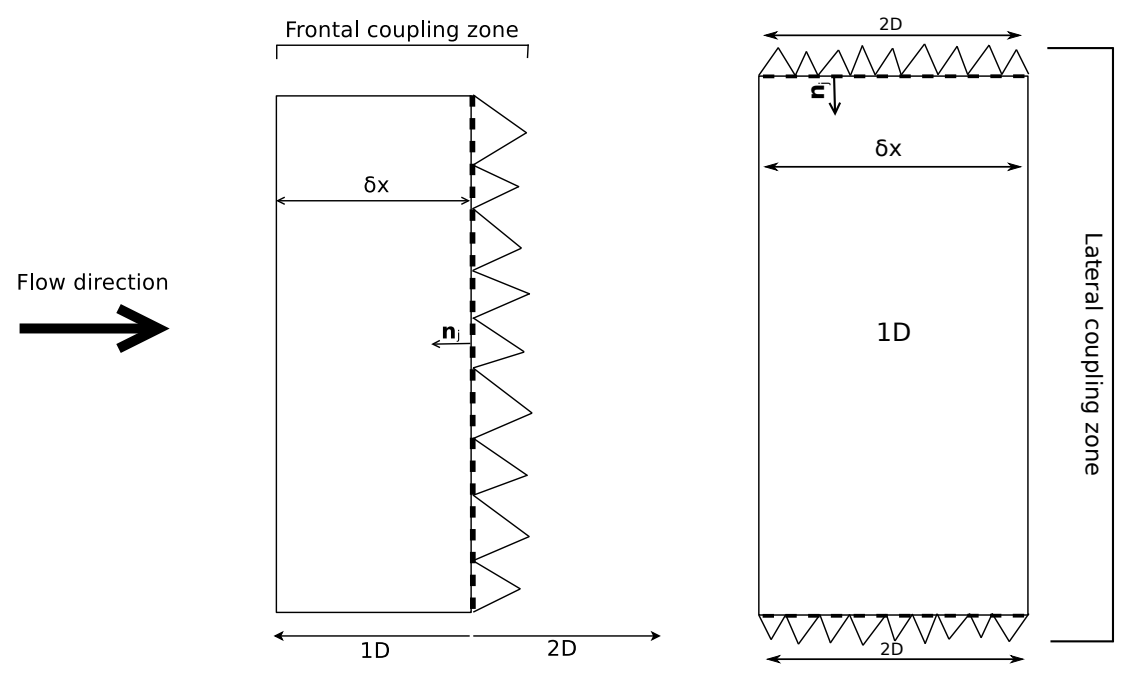

Figure 4: Frontal and lateral coupling zones 


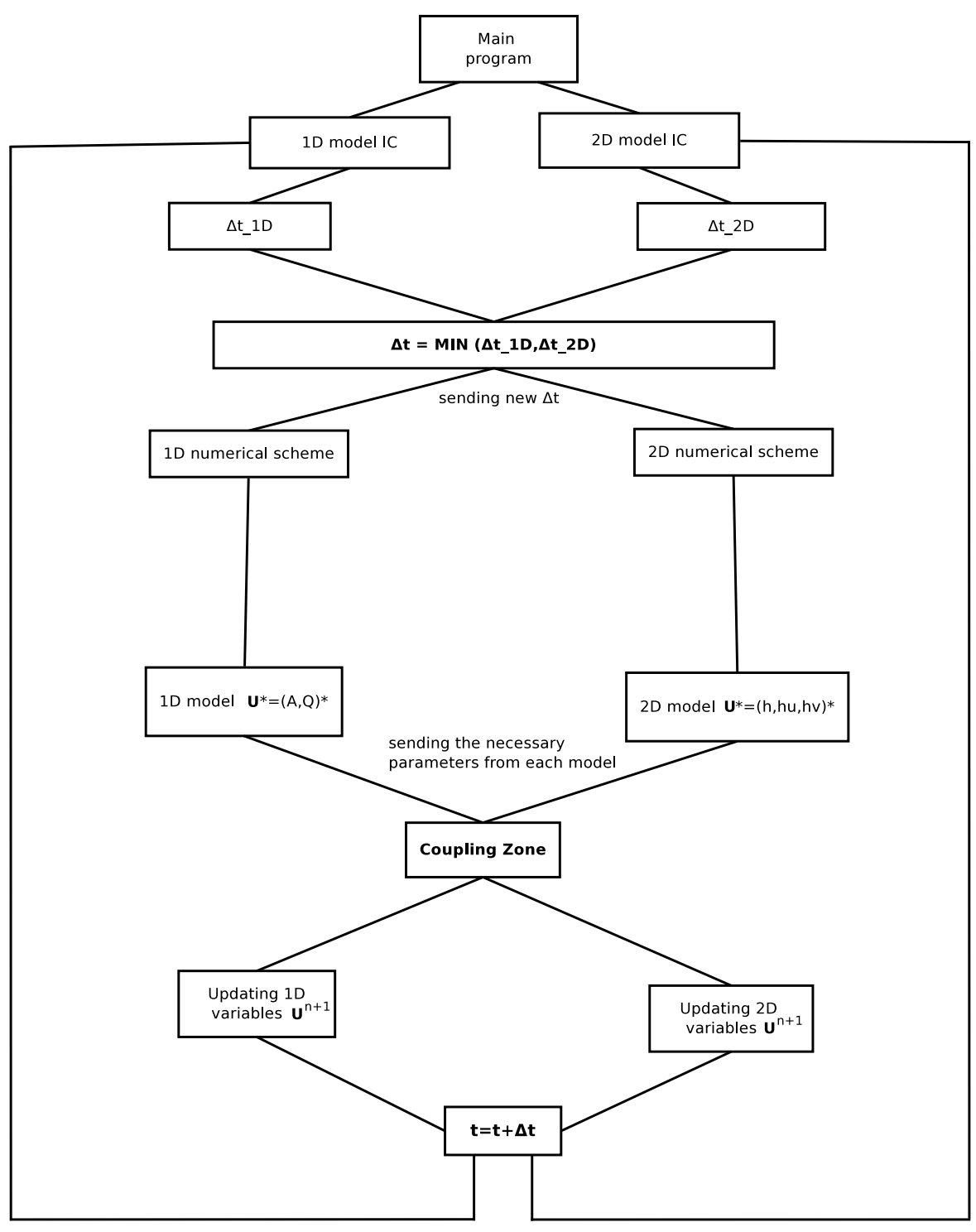

Figure 5: Flowchart of the coupled scheme 

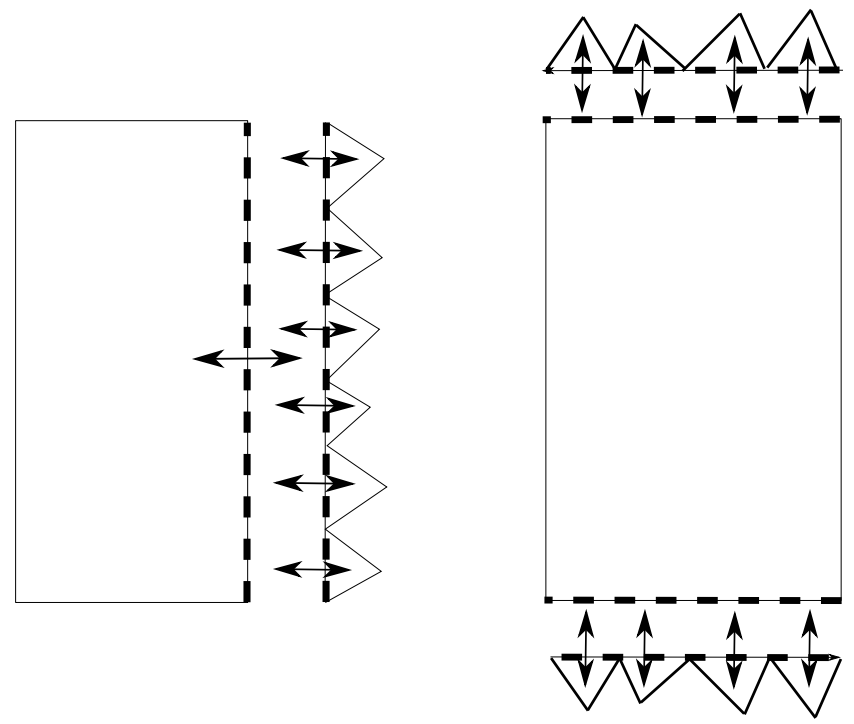

Figure 6: Contributions to be considered in mass conservation: frontal coupling (left) and lateral coupling (right)

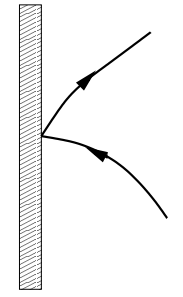

a)

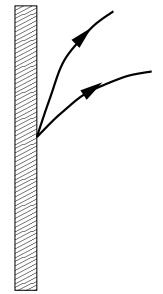

b)

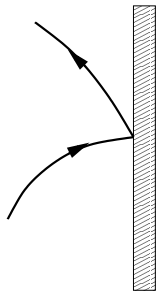

c)

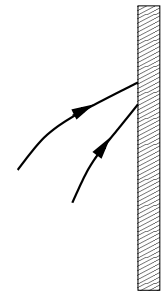

d)

Figure 7: Type of open boundaries: a) Subcritical inlet, b) Supercritical inlet, c) Subcritical outlet, d) Supercritical outlet 


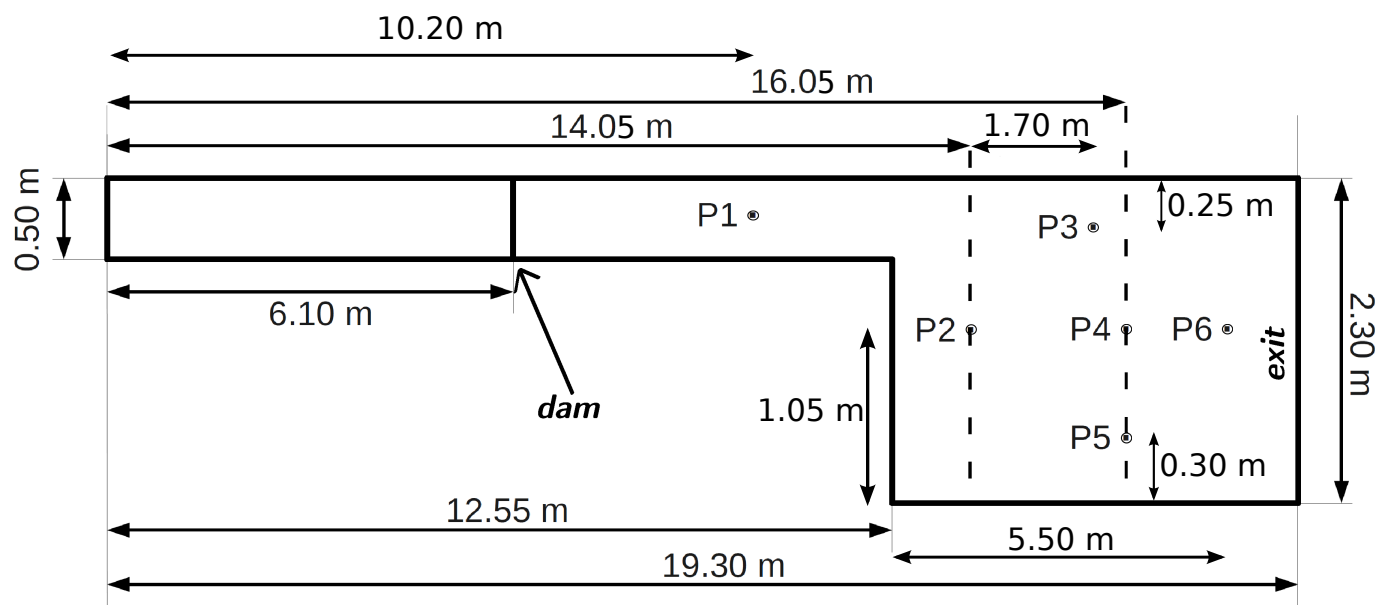

Figure 8: Description of the test case 1. Geometrical data. Position of the gauge points

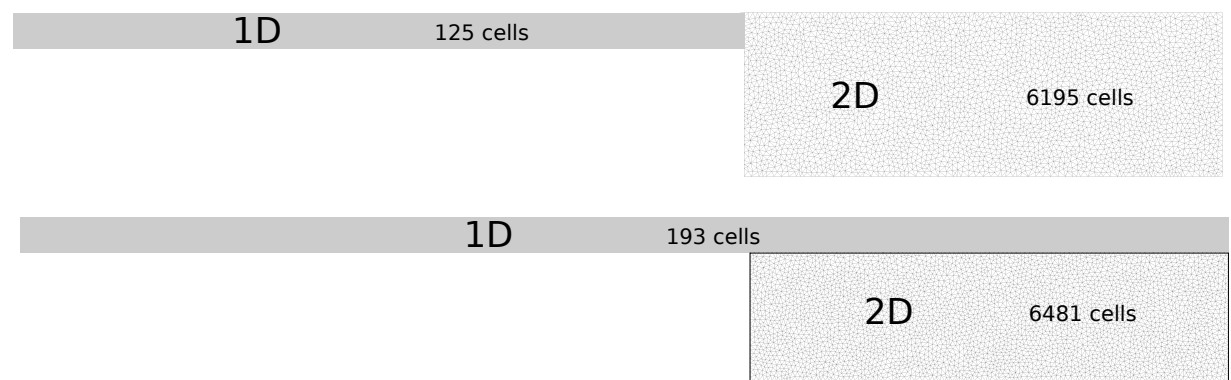

Figure 9: Test case 1: Upper: discretization of the frontal coupling domain. Lower: discretization of the lateral coupling domain 

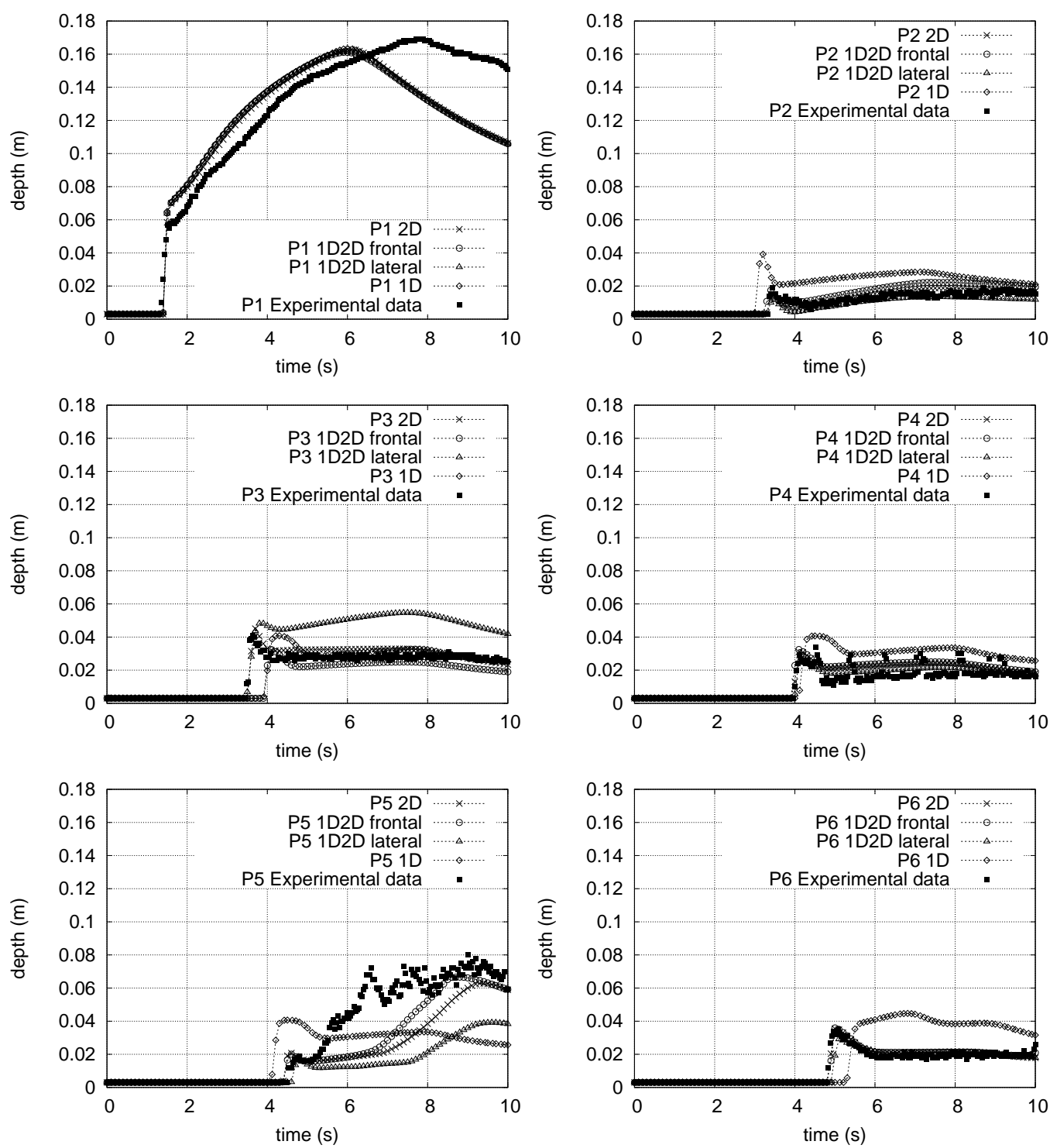

Figure 10: Test case 1: Comparison of numerical results and experimental measurements for the water depth at the gauge point P1 to P6, from upper left to lower down respectively 

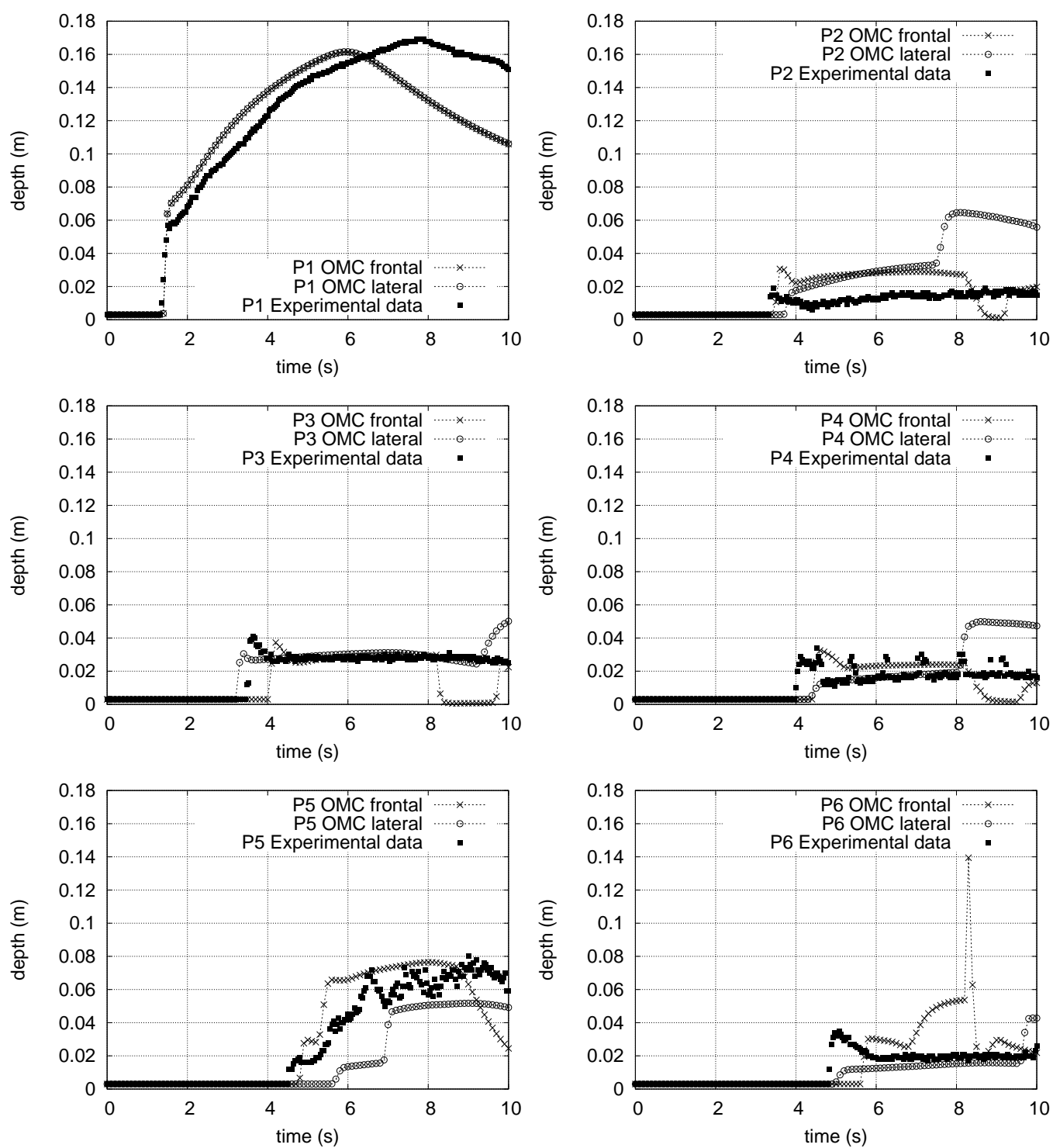

Figure 11: Test case 1: Comparison of numerical results with an $O M C$ strategy and experimental measurements for the water depth at the gauge point $\mathrm{P} 1$ to $\mathrm{P} 6$, from upper left to lower down respectively 


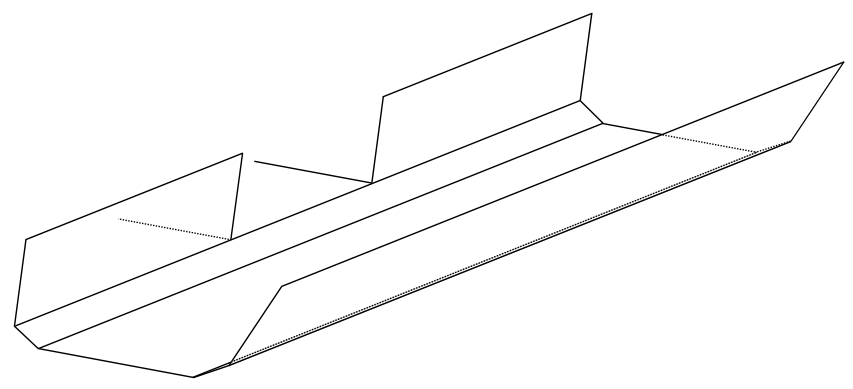

Figure 12: Description of the test case 2: a channel connected laterally with a floodplain area

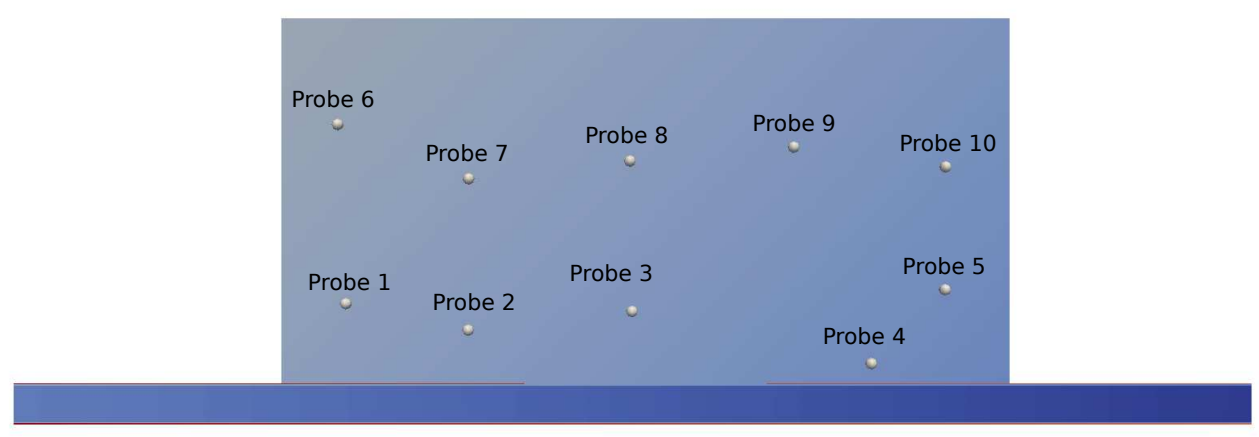

Figure 13: Test case 2: Position of the probes
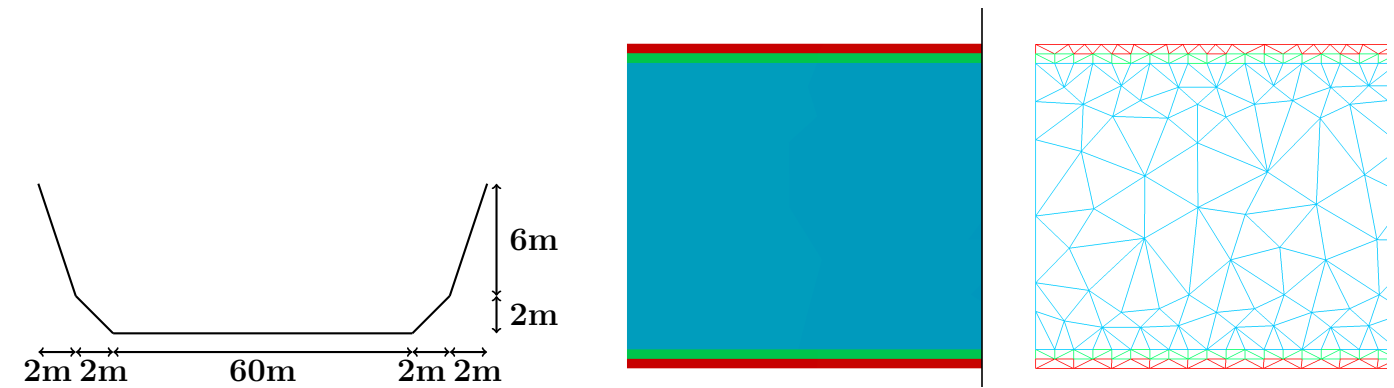

Figure 14: Test case 2: channel cross section geometry and 2D discretization into triangular cells 


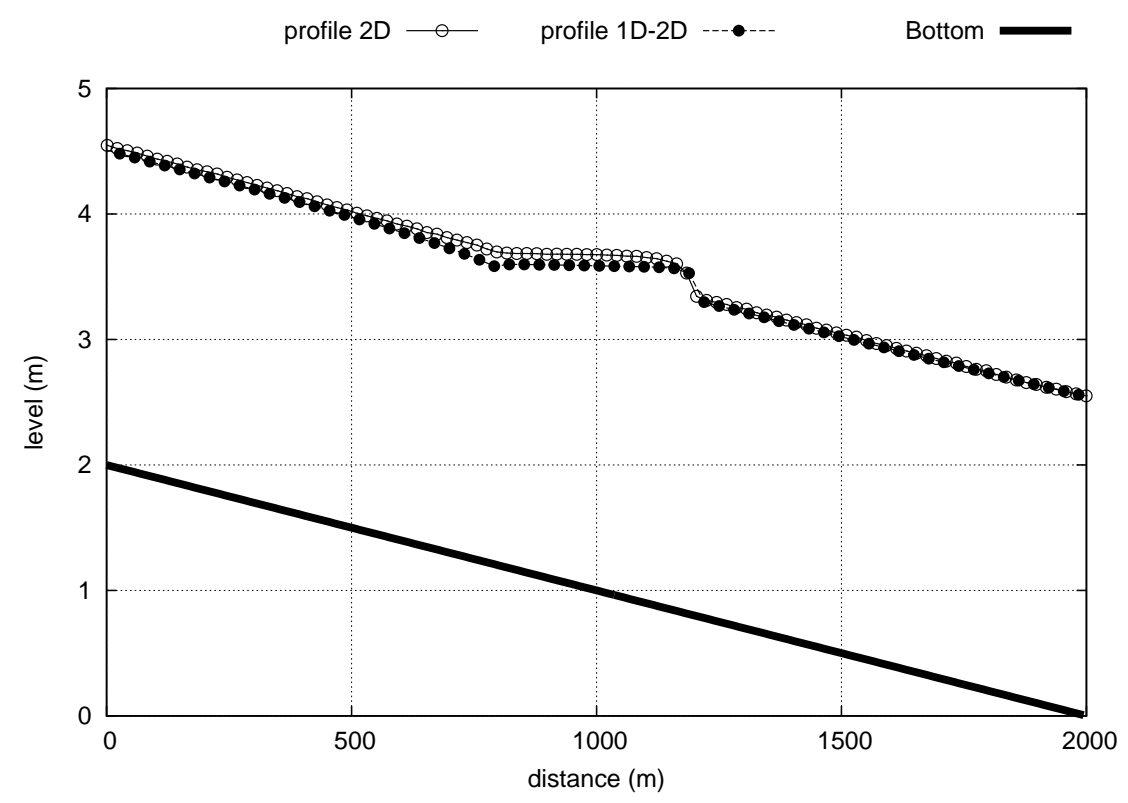

Figure 15: Test case 2 steady flow: longitudinal profile along the channel

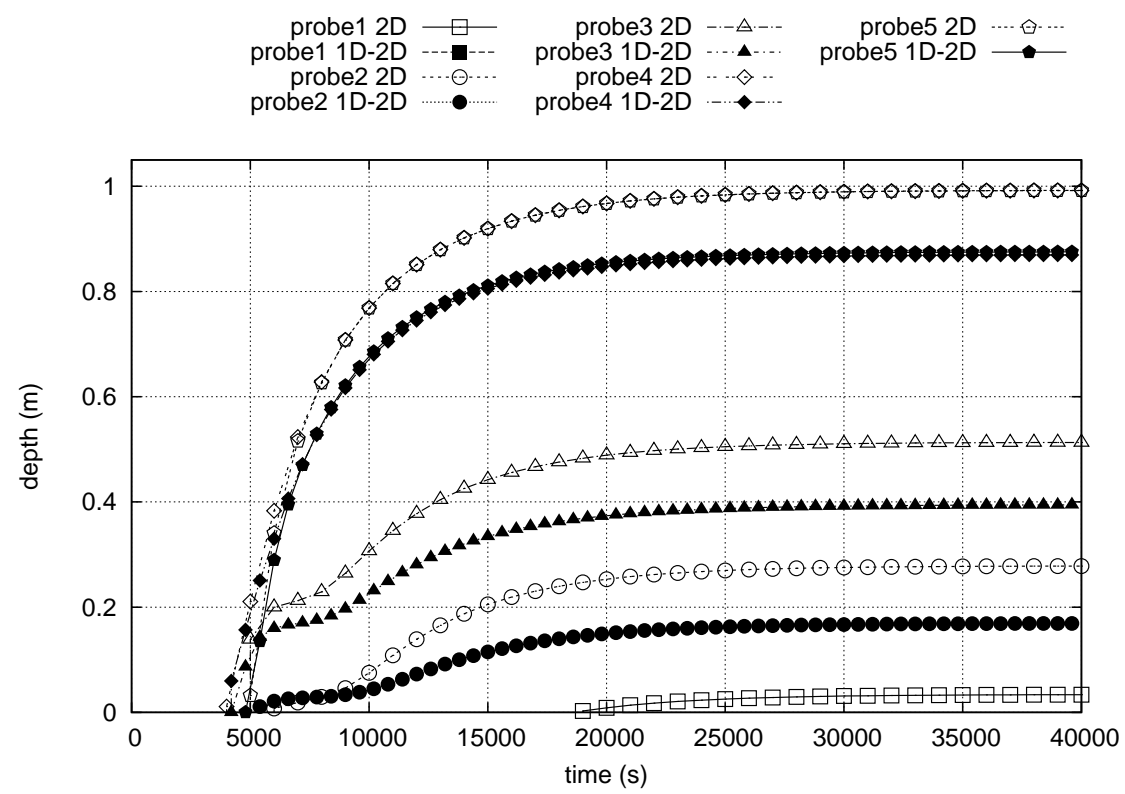

Figure 16: Test case 2 steady flow: probes 1-5. 1D-2D (shadow symbols), fully 2D (empty symbols) 


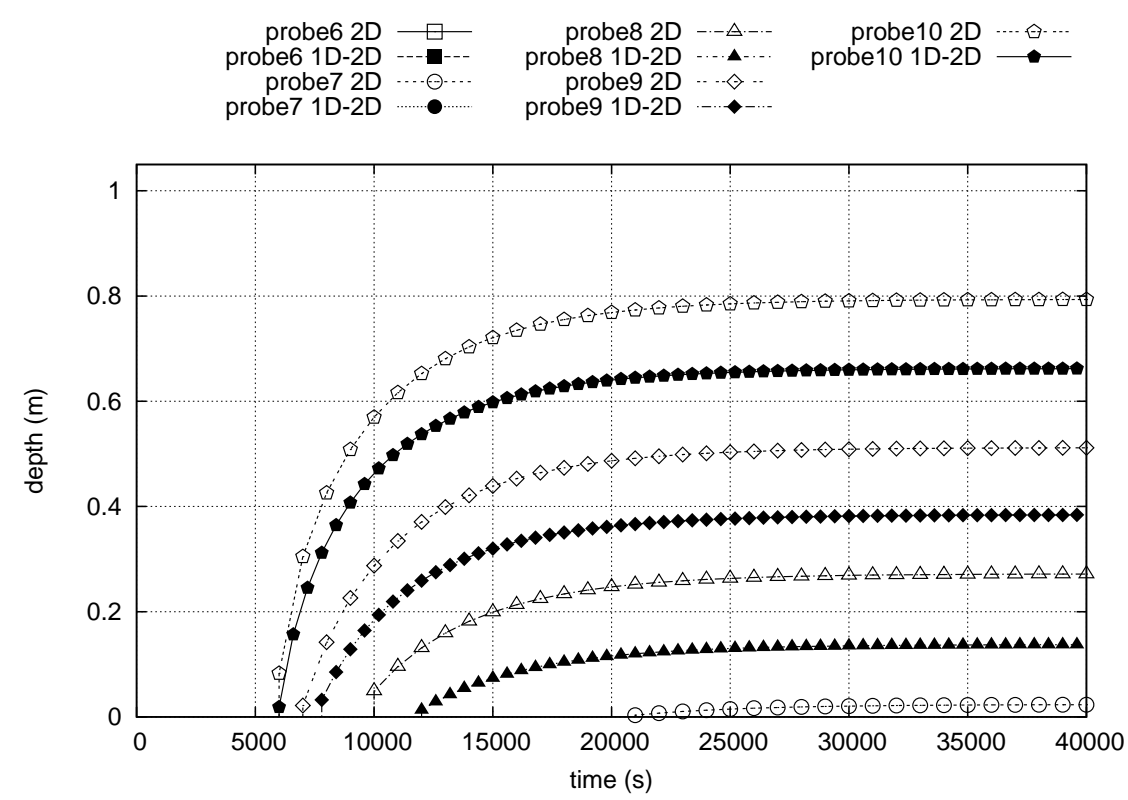

Figure 17: Test case 2 steady flow: probes 6-10. 1D-2D (shadow symbols), fully 2D (empty symbols)

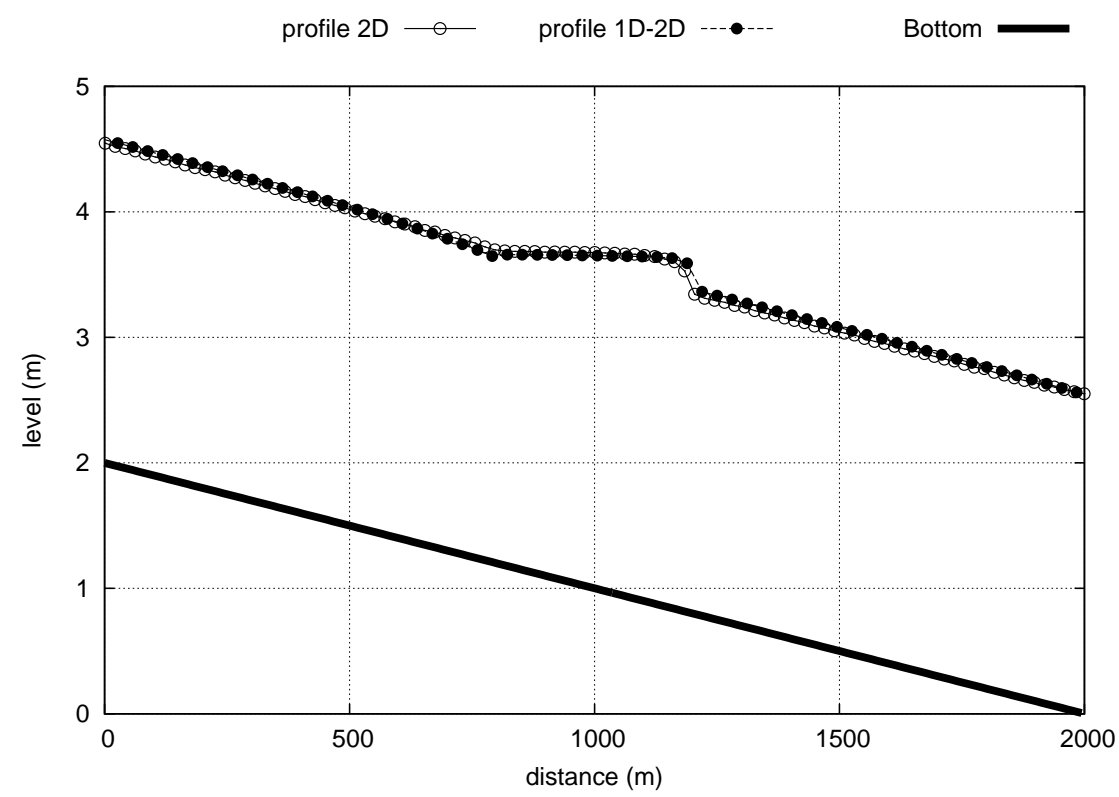

Figure 18: Test case 2 steady flow: longitudinal profile along the channel 


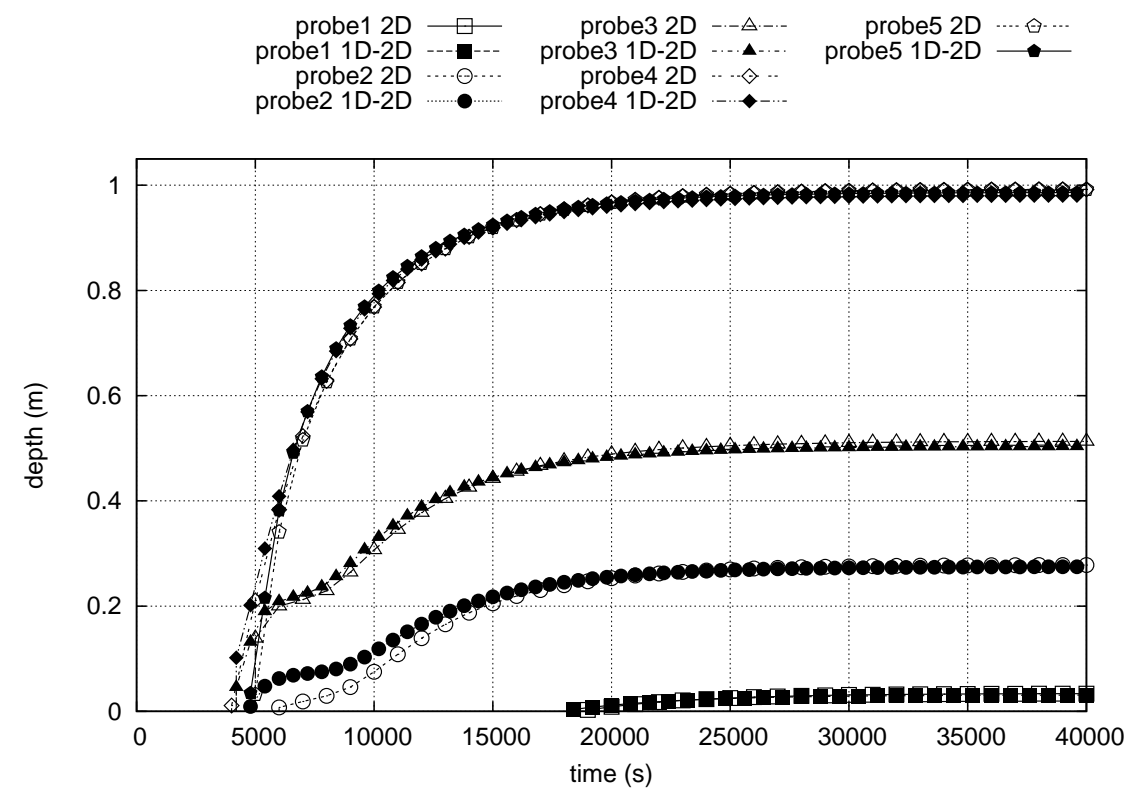

Figure 19: Test case 2 steady flow: probes $1-5 . \mathrm{n}=0.01605 \mathrm{~s} / \mathrm{m}^{\frac{1}{3}}$ in the river bed. 1D-2D (shadow symbols), fully 2D (empty symbols)

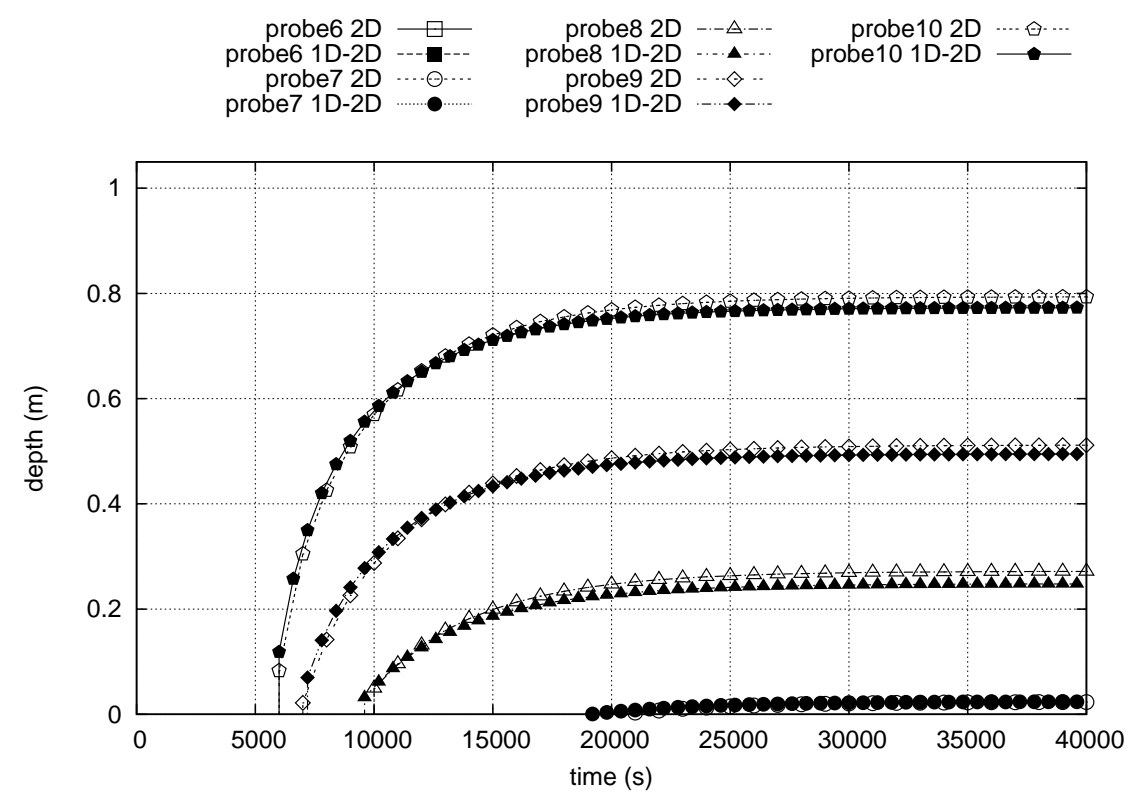

Figure 20: Test case 2 steady flow: probes $6-10 . n=0.01605 s / m^{\frac{1}{3}}$ in the river bed. $1 \mathrm{D}-2 \mathrm{D}$ (shadow symbols), fully 2D (empty symbols) 


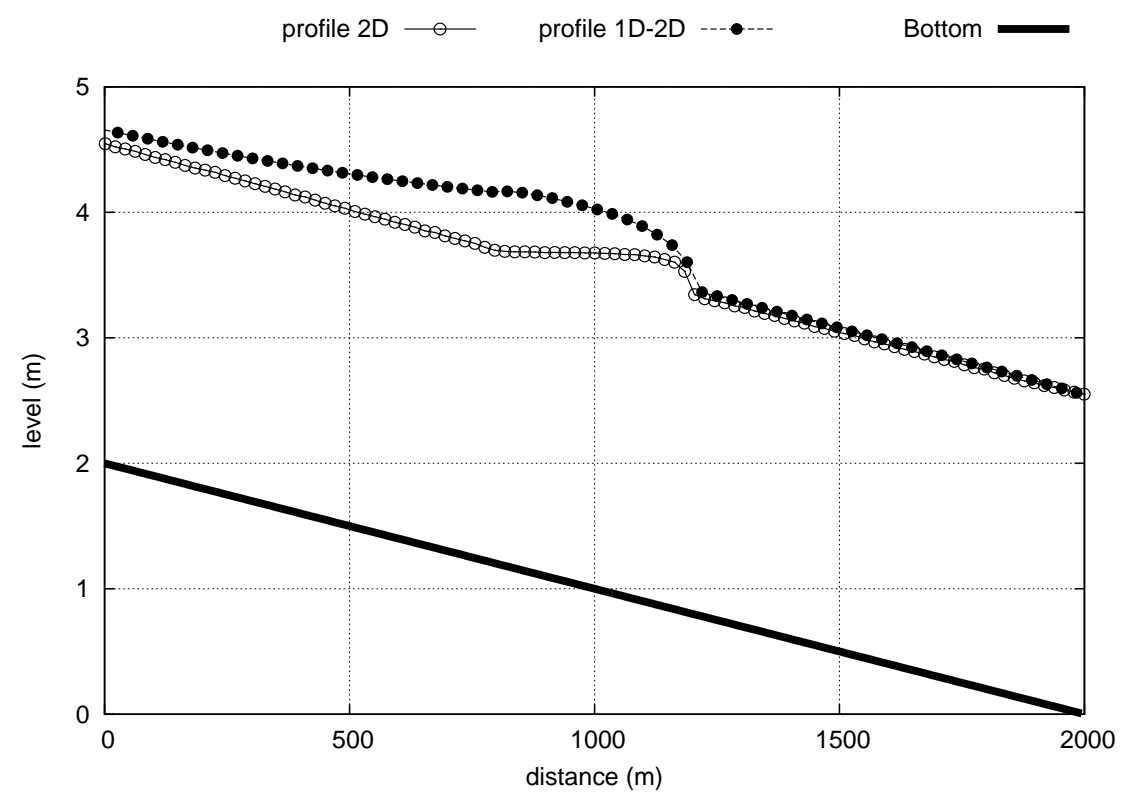

Figure 21: Test case 2 steady flow: longitudinal profile along the channel. $M M C$ strategy

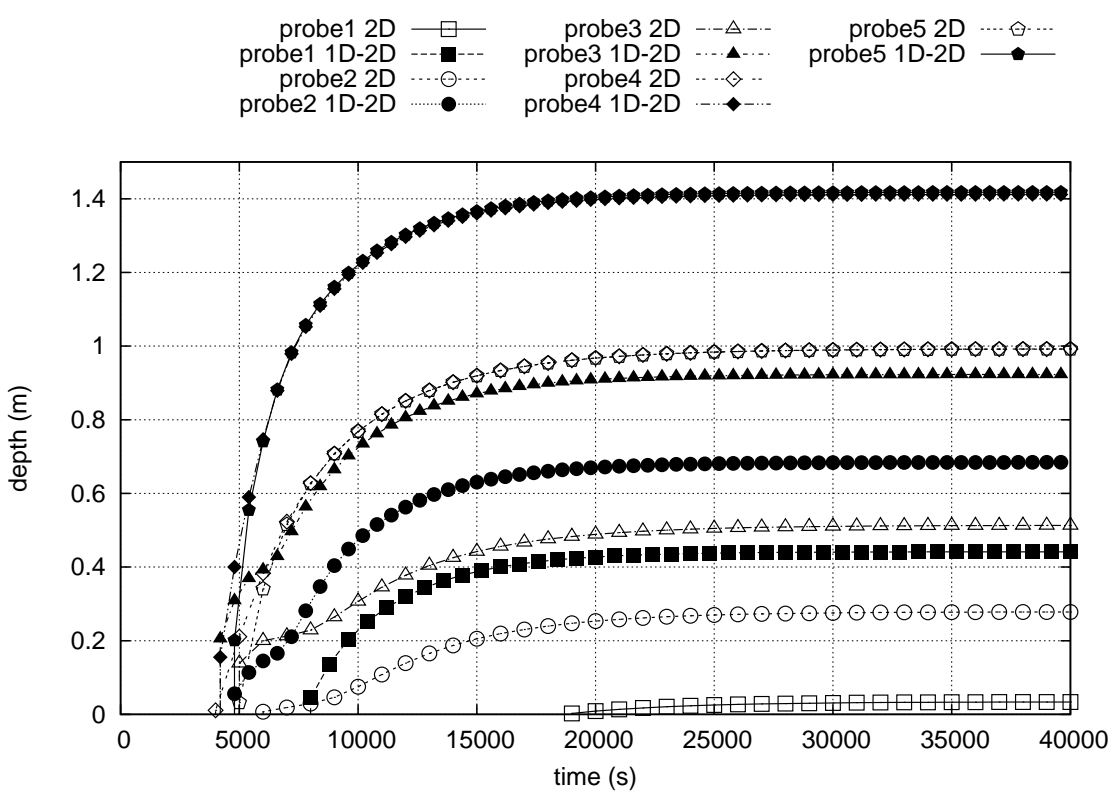

Figure 22: Test case 2 steady flow: probes $1-5 . \mathrm{n}=0.01605 \mathrm{~s} / \mathrm{m}^{\frac{1}{3}}$ in the river bed. $M M C$ strategy. 1D-2D (shadow symbols), fully 2D (empty symbols) 


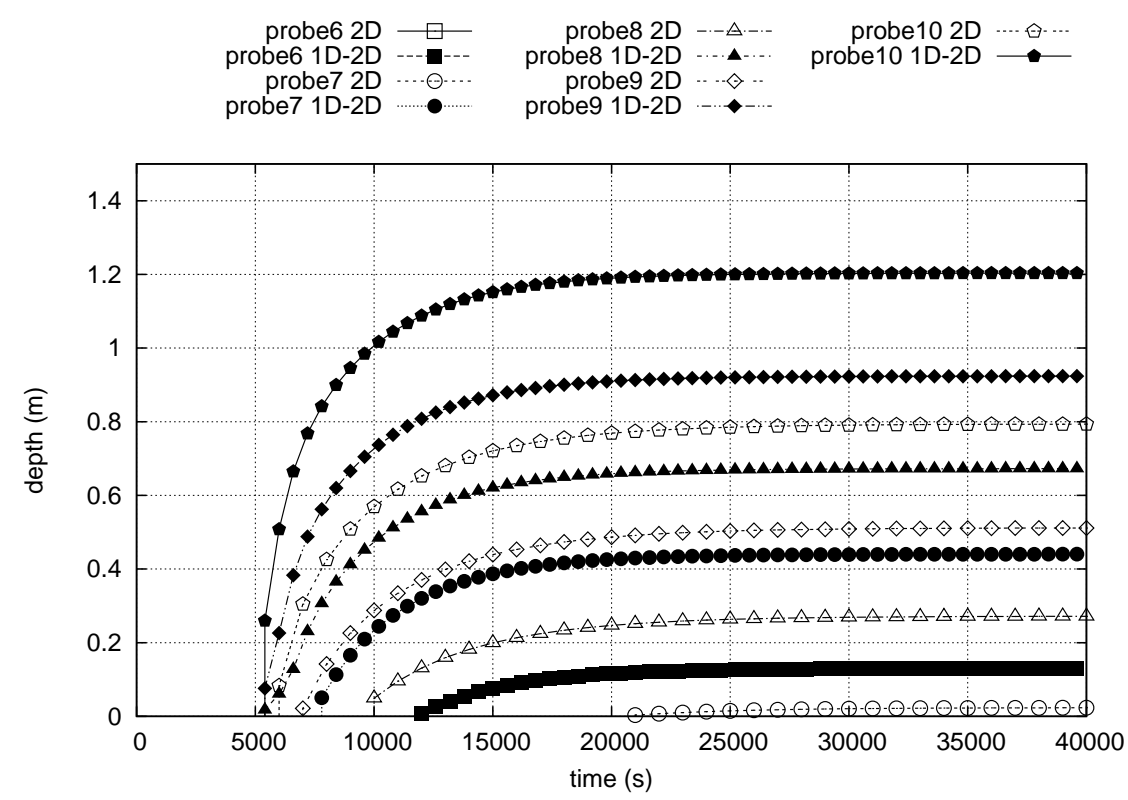

Figure 23: Test case 2 steady flow: probes $6-10 . \mathrm{n}=0.01605 \mathrm{~s} / \mathrm{m}^{\frac{1}{3}}$ in the river bed. $M M C$ strategy. 1D-2D (shadow symbols), fully 2D (empty symbols)

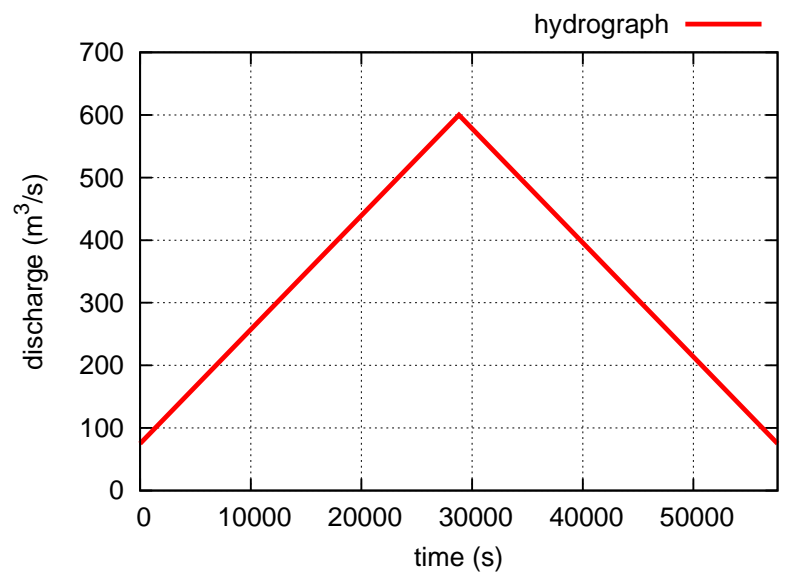

Figure 24: Test case 2: Triangular inlet hydrograph 


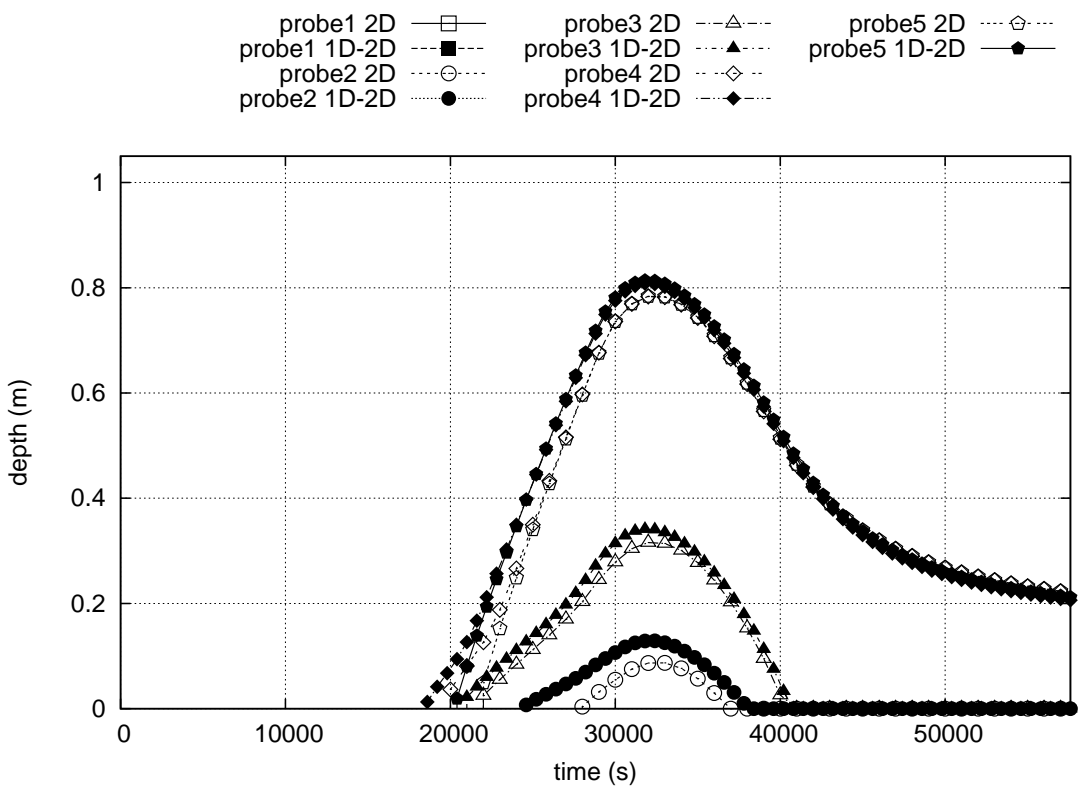

Figure 25: Test case 2 unsteady flow: probes 1-5. 1D-2D (shadow symbols), fully 2D (empty symbols)

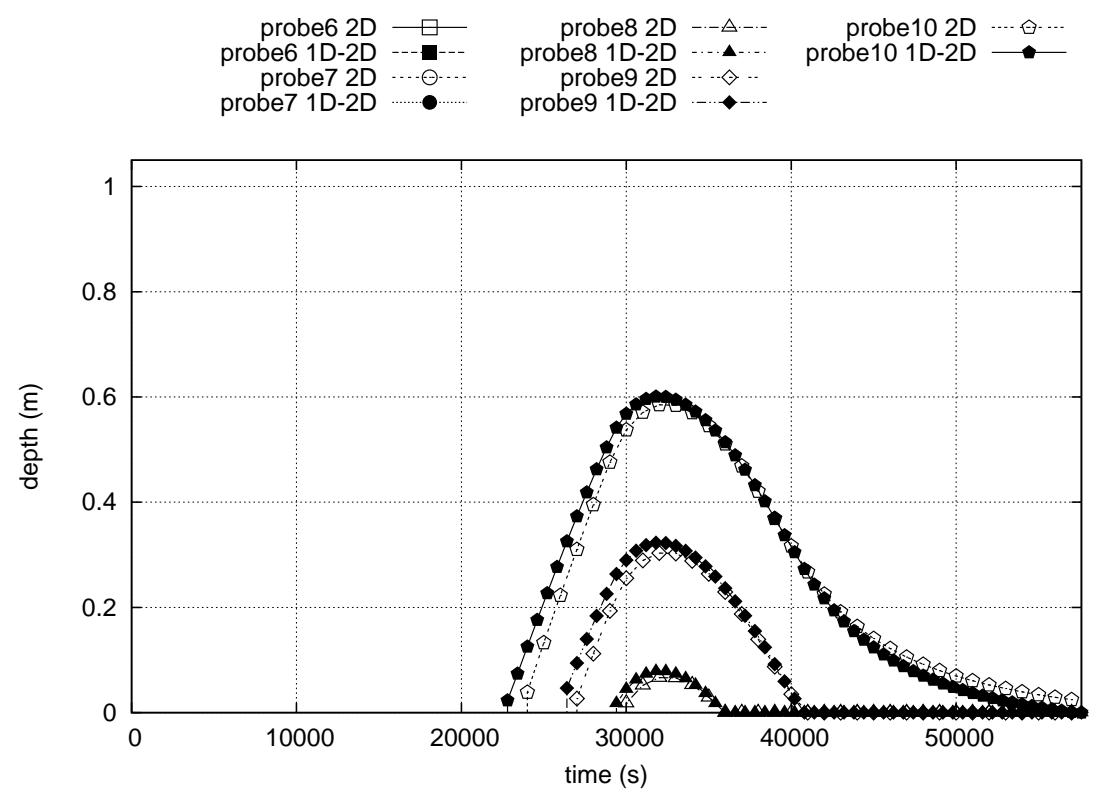

Figure 26: Test case 2 unsteady flow: probes 6-10. 1D-2D (shadow symbols), fully 2D (empty symbols) 


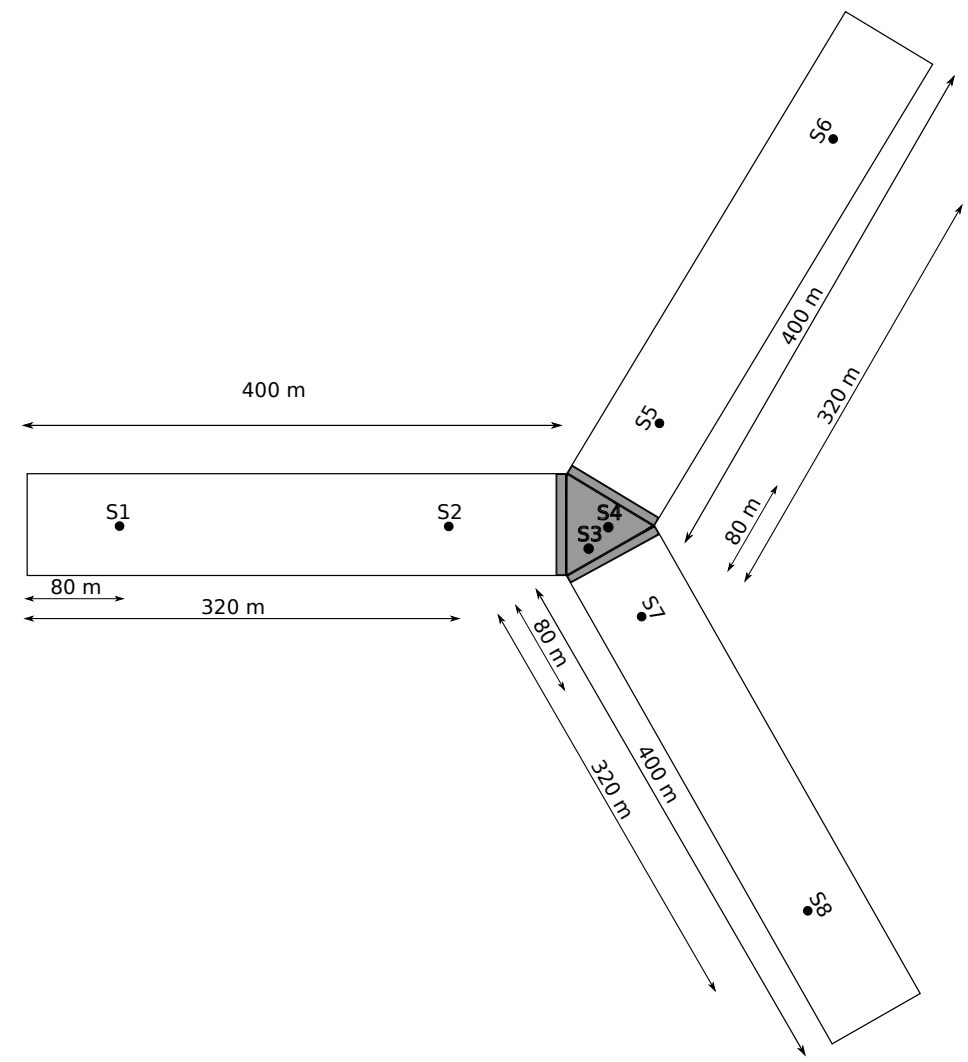

Figure 27: Description of the test case 3. Location of the gauge points. Plain background: 1D zone in the coupled model. Gray background: 2D zone in the coupled model 

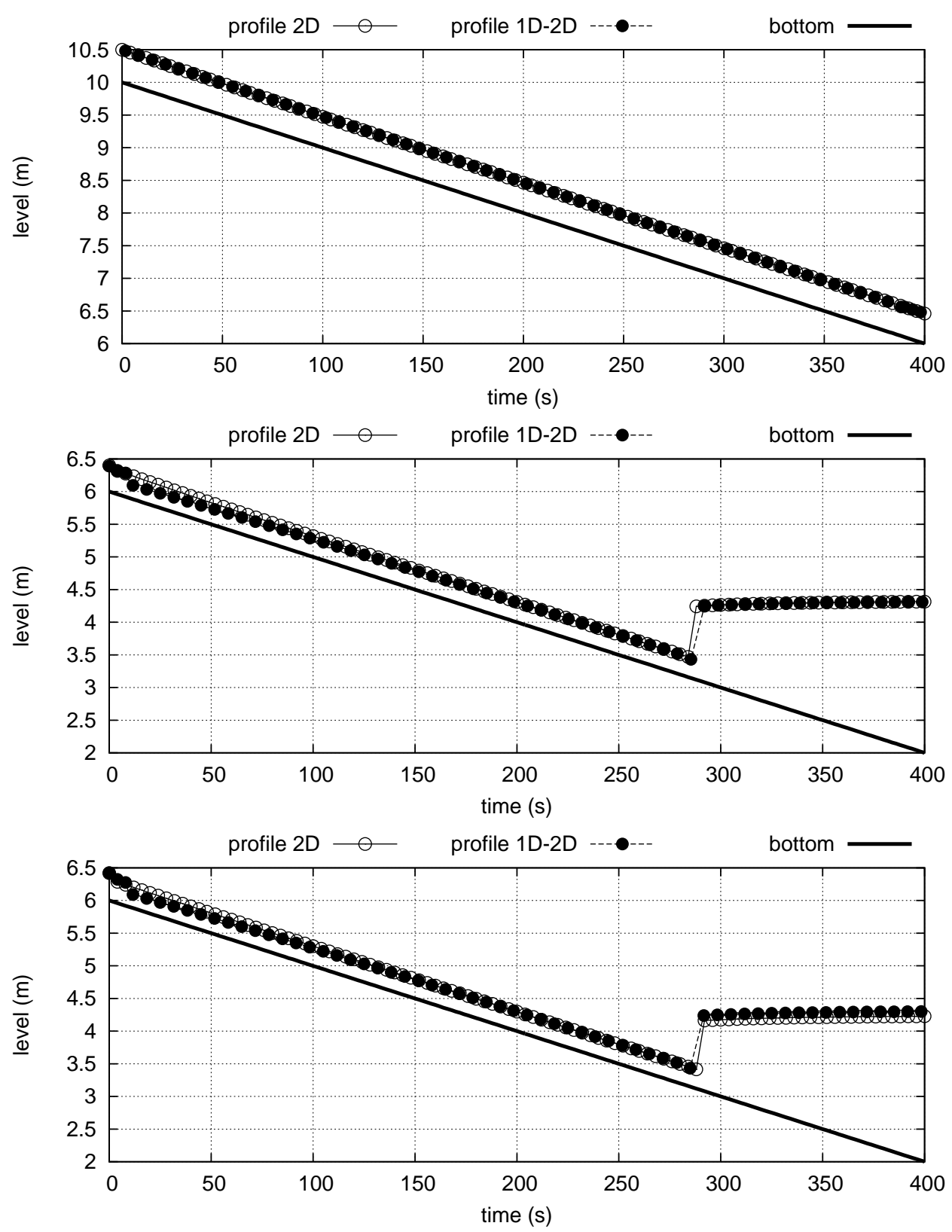

Figure 28: Test case 3: Longitudinal profiles of channel 1 (upper), channel 2 (intermediate) and channel 3 (lower). Supercritical junction 


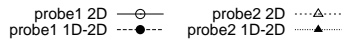
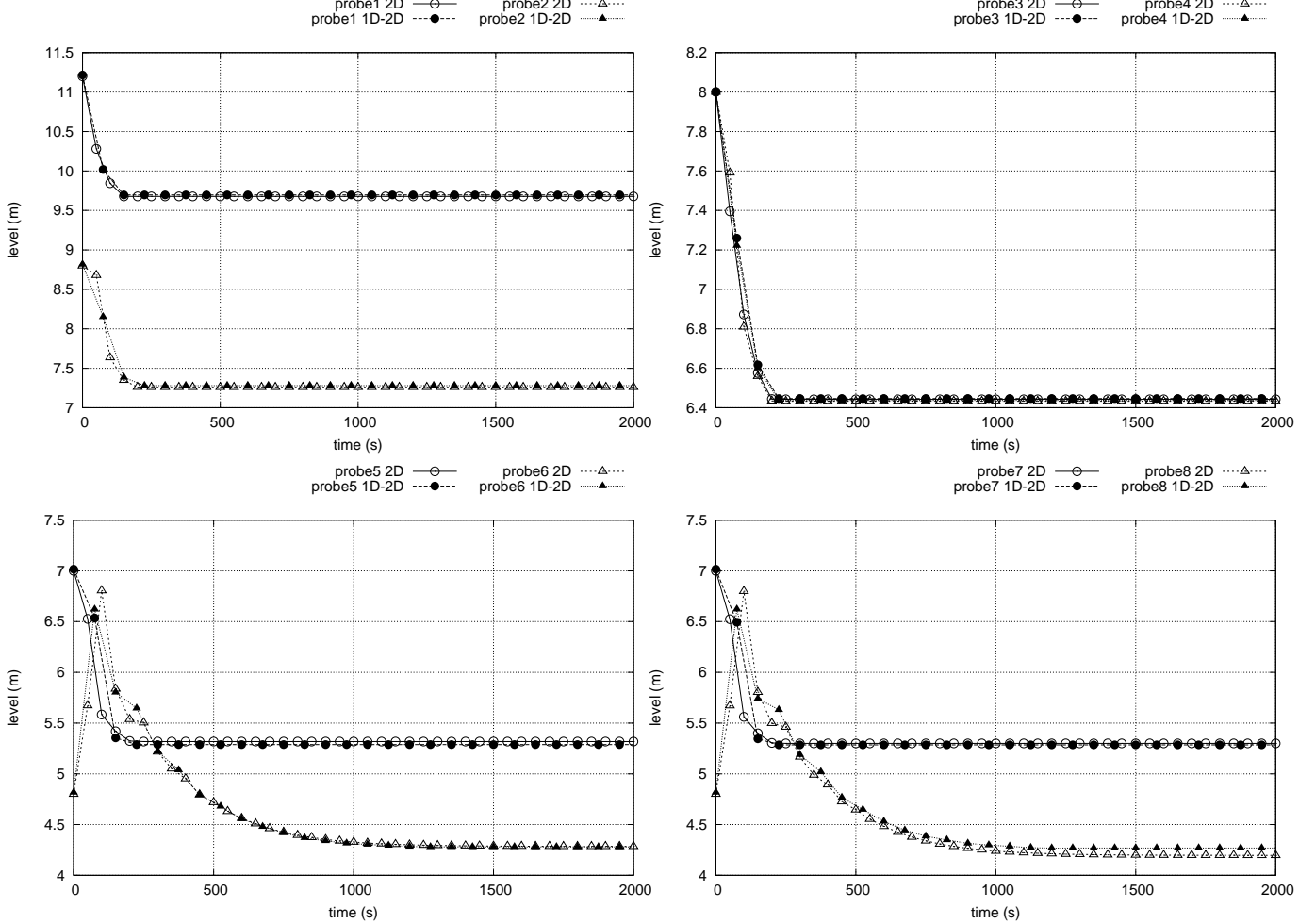

Figure 29: Test case 3: Comparison in terms of water level surface between the coupled model and the fully 2D model at each probe. Supercritical junction. 1D-2D (shadow symbols), fully 2D (empty symbols) 


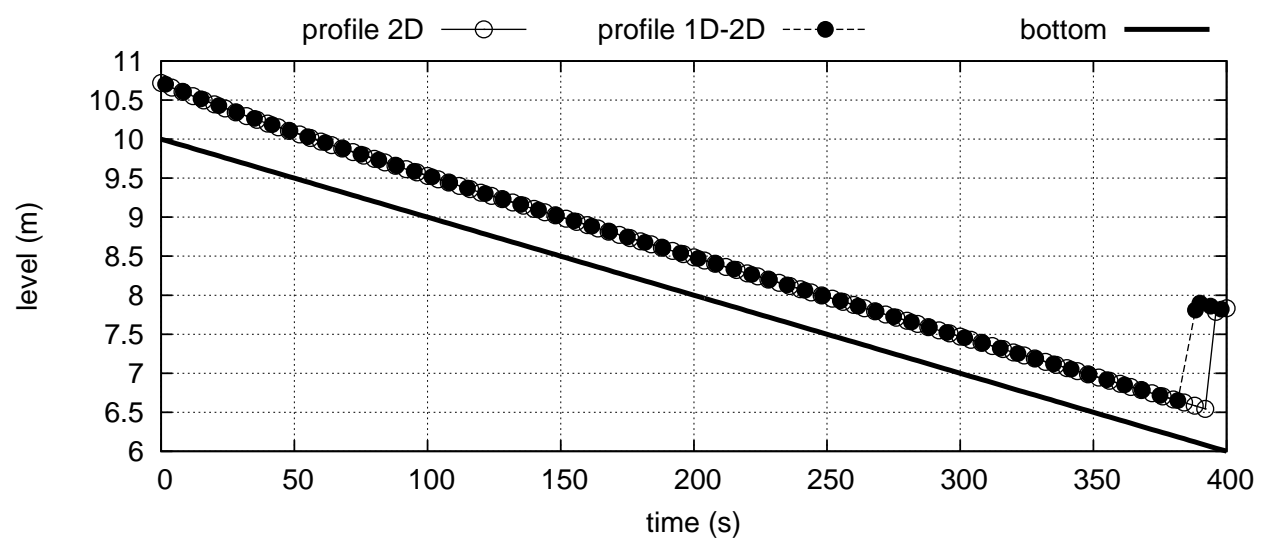

profile 2D $\bigcirc \quad$ profile 1D-2D --- $\bullet--$

bottom

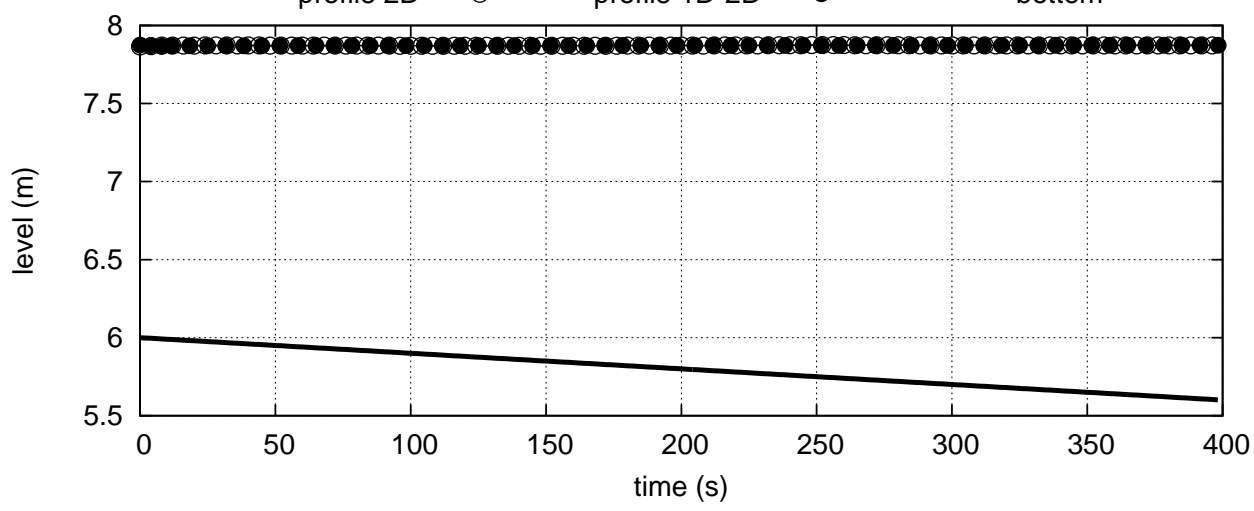

profile 2D $-0 \quad$ profile 1D-2D -----

bottom

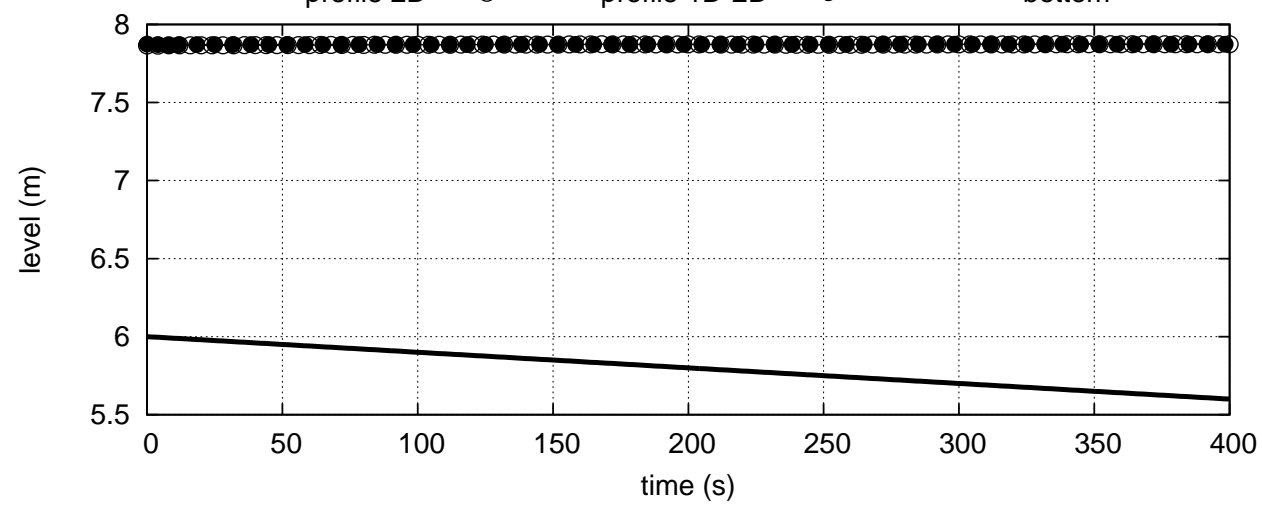

Figure 30: Test case 3: Longitudinal profiles of channel 1 (upper), channel 2 (intermediate) and channel 3 (lower). Subcritical junction 


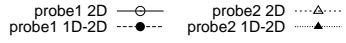

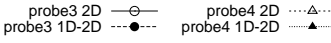
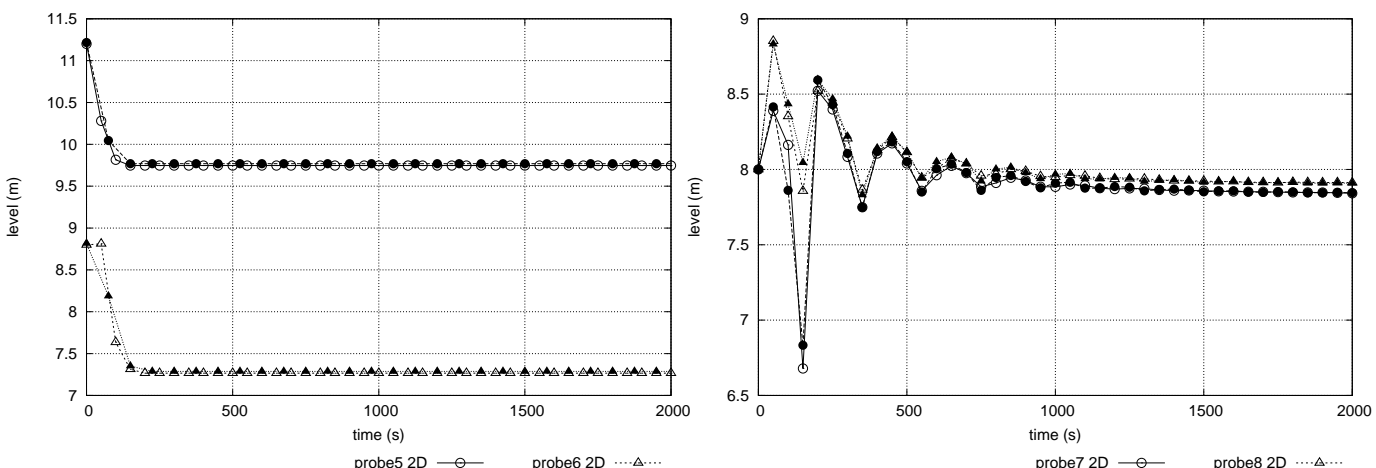

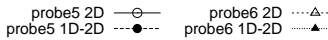

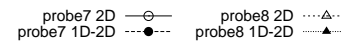
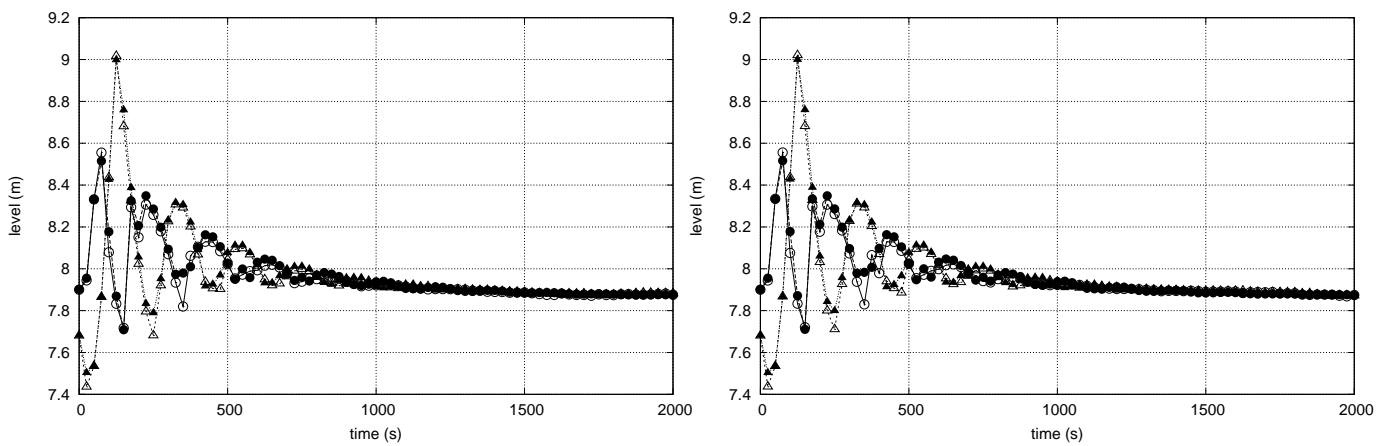

Figure 31: Test case 3: Comparison in terms of water level surface between the coupled model and the fully 2D model at each probe. Subcritical junction. 1D-2D (shadow symbols), fully 2D (empty symbols) 


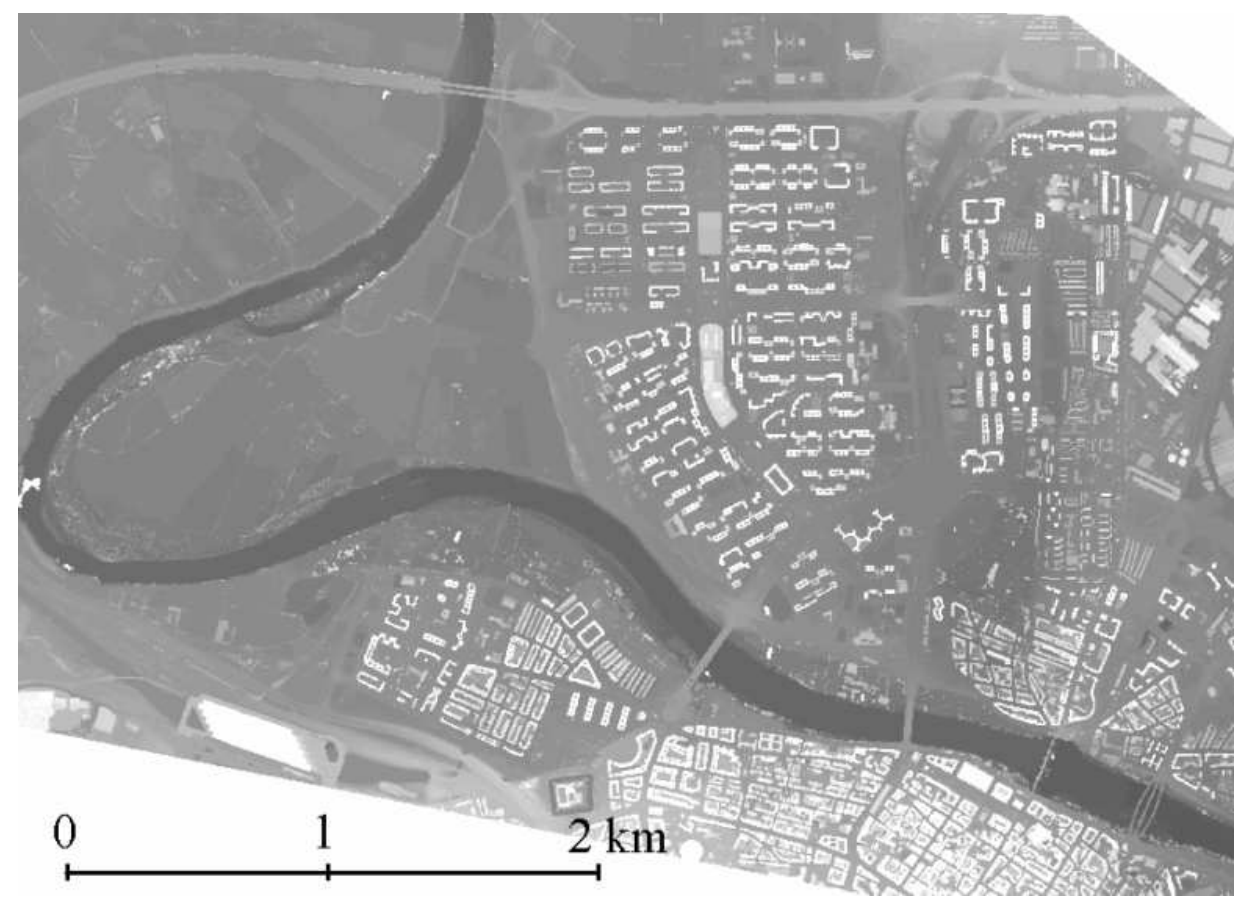

Figure 32: Description of the test case 4
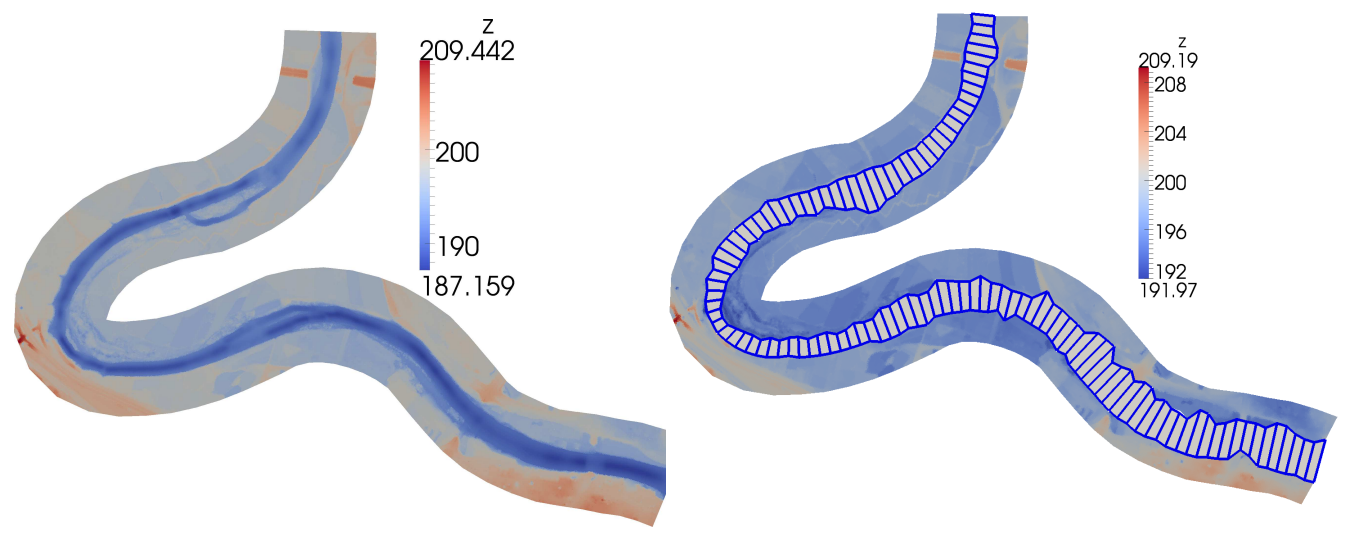

Figure 33: Test case 4: 2D model (left) and 1D-2D coupled model (right) for the Ebro river reach 


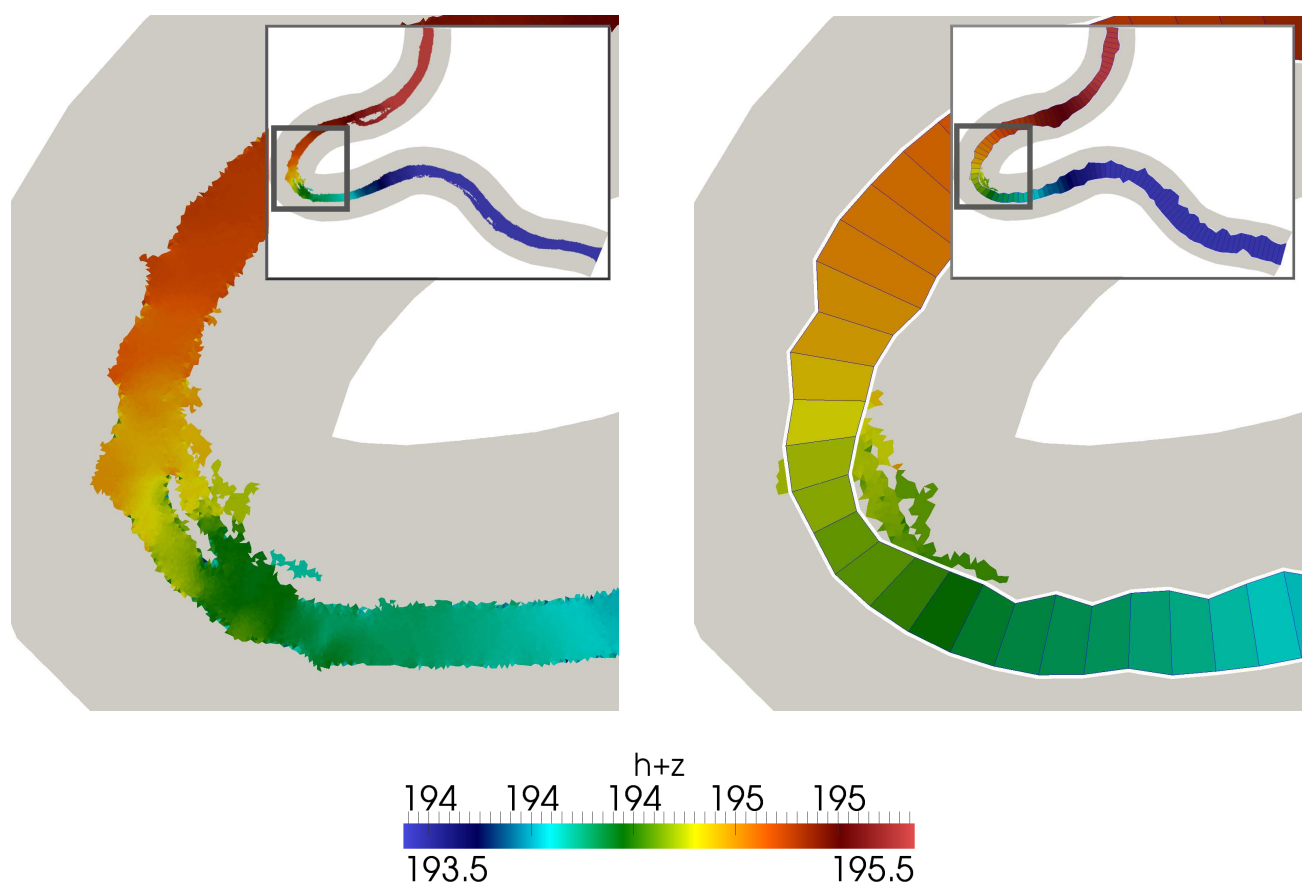

Figure 34: Test case 4: Numerical simulation of a steady flow of $600 \mathrm{~m}^{3} / \mathrm{s}$ in the Ebro river. Flooded area in the fully 2D model (left) and in the 1D-2D coupled model (right)

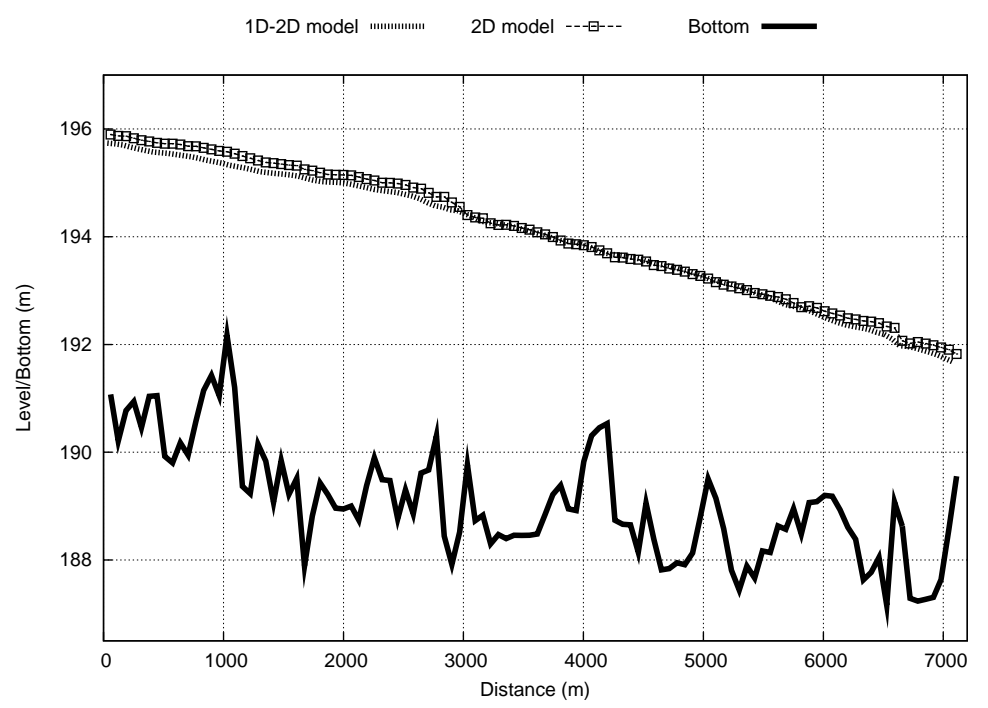

Figure 35: Test case 4: longitudinal profile along the river bed for the steady case 

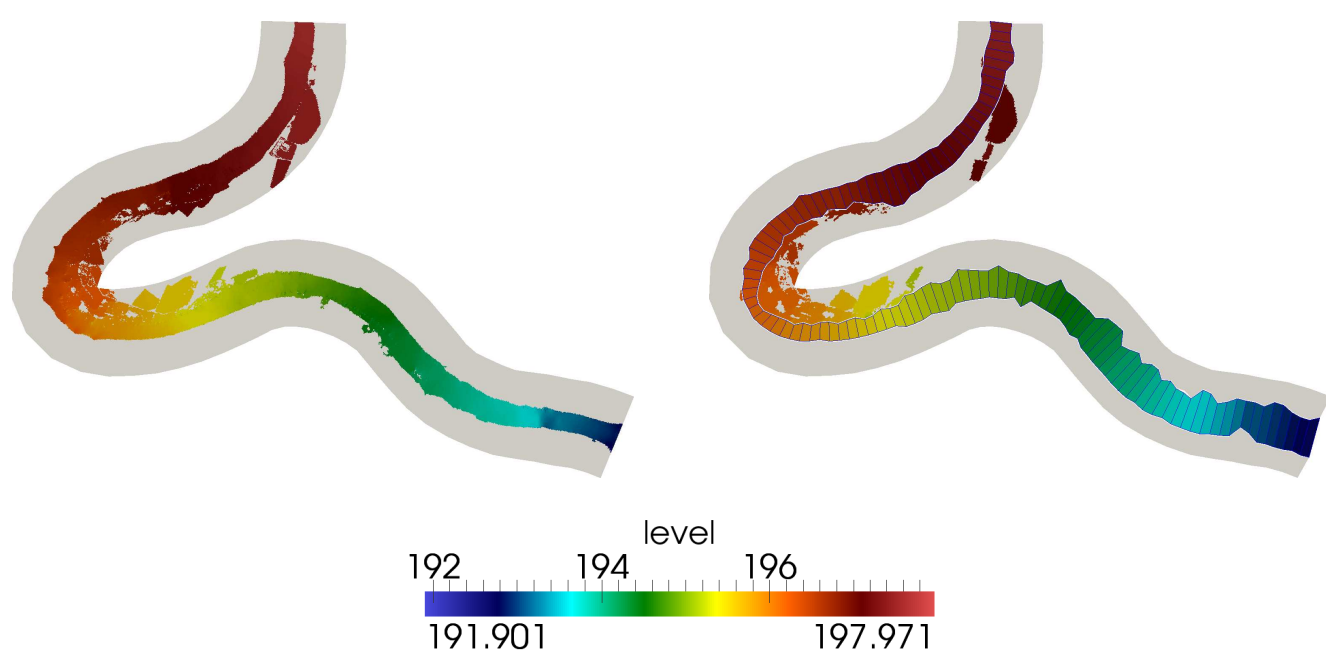

Figure 36: Test case 4: Numerical simulation of a unsteady flow in the Ebro river. Flooded area in the fully 2D model (left) and in the $1 \mathrm{D}-2 \mathrm{D}$ coupled model (right) at $\mathrm{t}=50000 \mathrm{~s}$

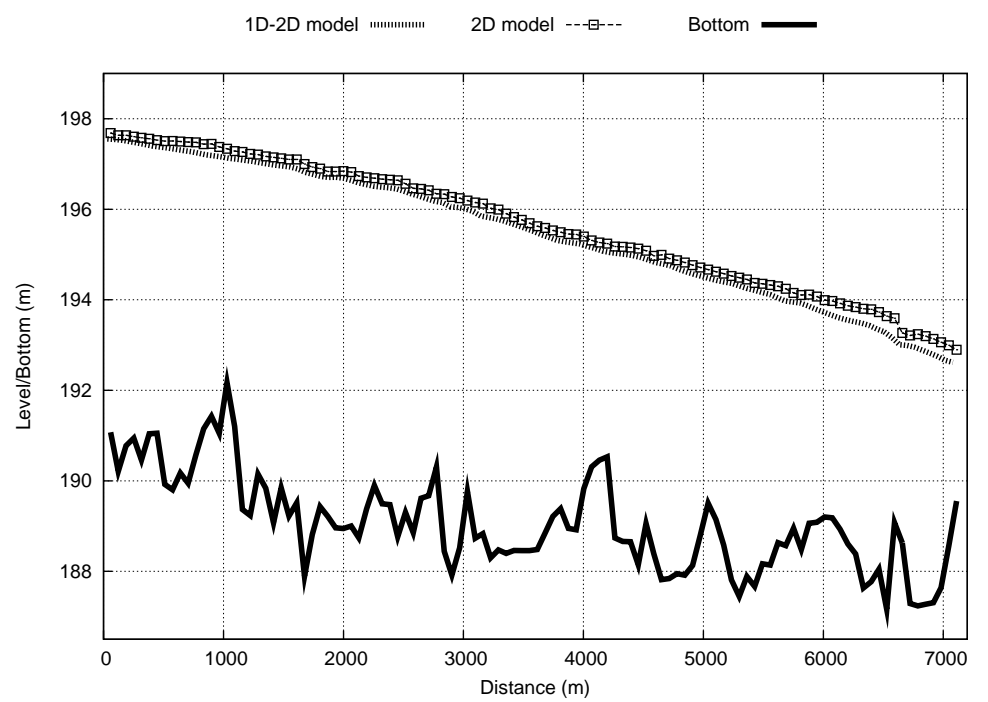

Figure 37: Test case 4: longitudinal profile along the river bed for the unsteady case at $\mathrm{t}=50000 \mathrm{~s}$ 


\begin{tabular}{c|c|c|c} 
& Flooded area 2D $\left(\mathrm{m}^{2}\right)$ & Flooded area 1D-2D $\left(\mathrm{m}^{2}\right)$ & Relative error \\
\hline Steady & $9.611965 \mathrm{e}+05$ & $9.829719 \mathrm{e}+05$ & $2.26 \%$ \\
\hline Unsteady & $1.4602498 \mathrm{e}+06$ & $1.4201994 \mathrm{e}+06$ & $2.74 \%$ \\
\hline & \multicolumn{3}{|c}{}
\end{tabular}

Table 1: Test case 4: Flooded area computed by the $1 \mathrm{D}-2 \mathrm{D}$ coupled model and the $2 \mathrm{D}$ model and relative error

\begin{tabular}{|c|c|c|c|c|}
\hline Test case & & Time $(s)$ fully $2 \mathrm{D}$ & Time $(s)$ 1D-2D coupled & Speed-up \\
\hline 1 & & 31 & 17 & 1.82 \\
\hline 2 & $\begin{array}{c}\text { Steady } \\
\text { Unsteady }\end{array}$ & $\begin{array}{l}66341 \\
11155 \\
\end{array}$ & $\begin{array}{c}2032 \\
376 \\
\end{array}$ & $\begin{array}{l}32.65 \\
29.66 \\
\end{array}$ \\
\hline 3 & $\begin{array}{c}\text { Supercritical } \\
\text { Subcritical }\end{array}$ & $\begin{array}{l}38416 \\
32521 \\
\end{array}$ & $\begin{array}{c}966 \\
1013 \\
\end{array}$ & $\begin{array}{c}39.77 \\
32.1 \\
\end{array}$ \\
\hline 4 & $\begin{array}{c}\text { Steady } \\
\text { Unsteady }\end{array}$ & $\begin{array}{l}31952 \\
50368\end{array}$ & $\begin{array}{l}117 \\
439\end{array}$ & $\begin{array}{l}273.09 \\
114.73\end{array}$ \\
\hline
\end{tabular}

Table 2: CPU time consumed by the 2D model and the coupled model in each test case 


\section{Appendix A. Computation of the common level surface at the cou- pling zone}

The proposed 1D-2D coupled model is based on imposing a common water level surface at the coupling zone. The conscientiously technique explained before should also be combined with a carefully computation of the common level once the total water volume of the coupling zone, $V_{C Z}$, has been calculated. The aim consists of building a level-volume table for each coupling zone in the pre-process and, during the computation, to assign the corresponding level of such water volume.

Let consider a sliced sketch of a coupling zone as in Figure A.38. The irregular cross section represented by the 1D model in left side is connected through several short straight lines at the right side, representing the bottom or elevation of the 2D coupled cells.

First of all, it is necessary to sort the vector generated with all the levels involved at the coupling zone, which are represented by the dashed lines. By traversing from lower to higher surface level in this vector, a table with the information included in (A.1)

$$
\begin{array}{lllll}
z_{k} & b_{k} & S_{k} & Z_{k} & V_{k}
\end{array}
$$

must be built, where $k$ indicates the index, $z$ is the surface level, $b$ is the corresponding width in the $1 \mathrm{D}$ model, $S$ includes the accumulated $2 \mathrm{D}$ cell sizes, $Z$ is the corresponding side slopes and $V$ is the water volume. This 
volume is achieved following the algorithm (A.2):

$$
V_{k+1}=V_{k}+C_{k}\left(z_{k+1}-z_{k}\right)+\frac{1}{2} Z_{k} \delta x\left(z_{k+1}-z_{k}\right)^{2}
$$

being $\delta x$ the $1 \mathrm{D}$ cell size and $C=b \delta x+S$. During the computation, a water volume $V_{C Z}$ is calculated at the coupling zone, whose corresponding correct level $z_{s}^{n+1}$ will be imposed there. In order to do this assignment, the second order (in $z_{s}^{n+1}$ ) equation (A.3) should be solved:

$$
V_{C Z}=V_{j}+C_{j}\left(z_{s}^{n+1}-z_{j}\right)+\frac{1}{2} Z_{j} \delta x\left(z_{s}^{n+1}-z_{j}\right)^{2}
$$

where $j$ is the immediately lower index from that $V_{C Z}$ is located in the table. Finally, the only solution for $z_{s}^{n+1}$ which stays between $z_{j}$ and $z_{j+1}$ is imposed as the common water surface level.

It is important to remark that this technique provides good accuracy in the presence of irregular geometries, allowing to discern which are the $2 \mathrm{D}$ cells that must be wet when imposing a common level surface. 


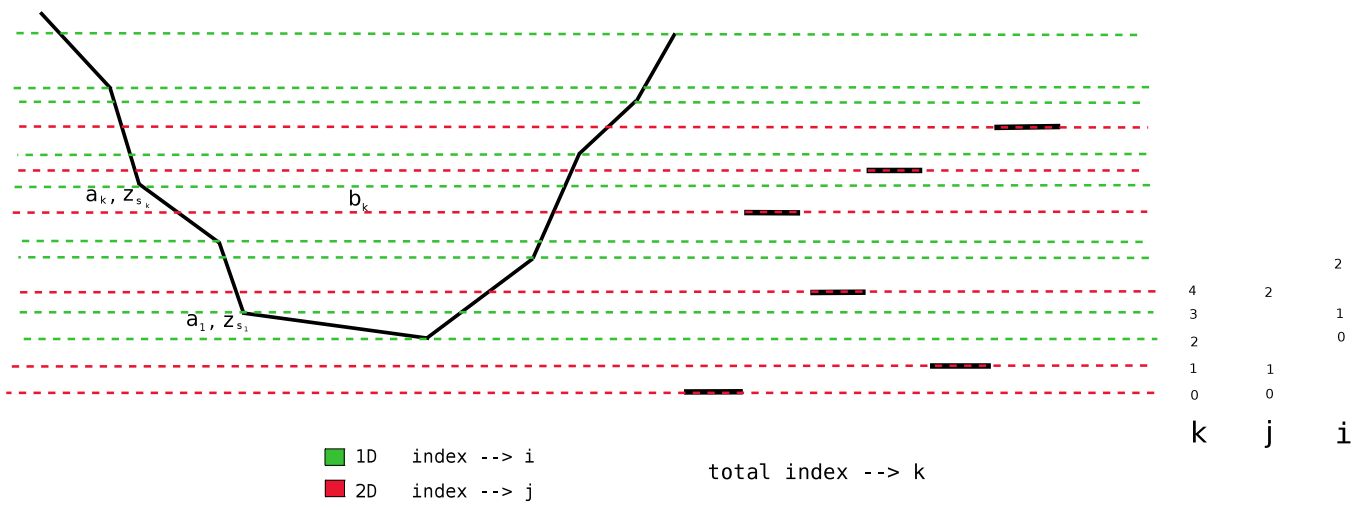

Figure A.38: Sliced sketch of a coupling zone 